$\operatorname{IMSC} / 2012 / 2 / 2$

\title{
Loop Variables and Gauge Invariant Exact Renormalization Group Equations for (Open) String Theory.
}

\author{
B. Sathiapalan \\ Institute of Mathematical Sciences \\ Taramani \\ Chennai, India 600113
}

August 23, 2018

\begin{abstract}
An exact renormalization group equation is written down for the world sheet theory describing the bosonic open string in general backgrounds. Loop variable techniques are used to make the equation gauge invariant. This is worked out explicitly up to level 3 . The equation is quadratic in the fields and can be viewed as a proposal for a string field theory equation. As in the earlier loop variable approach, the theory has one extra space dimension and mass is obtained by dimensional reduction. Being based on the sigma model RG, it is background independent. It is intriguing that in contrast to BRST string field theory, the gauge transformations are not modified by the interactions up to the level calculated. The interactions can be written in terms of gauge invariant field strengths for the massive higher spin fields and the non zero mass is essential for this. This is reminiscent of Abelian Born-Infeld action (along with derivative corrections) for the massless vector field, which is also written in terms of the field strength.
\end{abstract}




\section{Introduction}

The renormalization group has been applied to the world sheet action for a string propagating in non-trivial backgrounds to obtain equations of motion [1]-[14]]. One of the unsolved problems is to write down gauge invariant (under space-time gauge transformations) RG equations for all the modes of the string i.e. an exact renormalization group (ERG). This would then be equivalent to string field theory. The first systematic attempt to connect string field theory with the ERG was made in [9, 29].

A generalization of this technique involving Loop Variables has been used to give a partial solution to this problem [15, 18, 19]. The free equation were written down. A version of the interacting equations were also written down. There were a couple of noteworthy features : The interacting equations were made to look exactly like the free equations (by employing the OPE and Taylor expansion in the presence of a finite cutoff), and thus the mechanism of gauge invariance was very similar to that of the free theory. This is conceptually interesting. However it turns out that written in terms of space time fields the gauge invariance of any equation involves contributions from all mass levels. This has the consequence that one can either make the gauge invariance manifest, or the space time field structure manifest. Furthermore it is necessary to sum an infinite series of terms before the continuum limit (on the world sheet) can be taken. Thus it has not been possible thus far to write down a finite consistently truncated set of equations in terms of space time fields where the gauge invariance is manifest.

In fact, in the approach to interactions advocated in [18, it is possible to make field redefinitions, at any given order, that make the (space time theory) theory look free. However these field redefinitions are singular when the cutoff is removed. This is not surprising: As long as the cutoff is finite there are no poles in the string S-Matrix and the theory is trivial. The poles arise when the continuum limit is taken and then the theory is not free. So the continuum limit is crucial and it is thus important to write the theory in a way that allows this limit to be taken.

The aim of this work is to write down a gauge invariant exact renormalization group (ERG). In the ERG the equations are quadratic. The free part is the same as in the earlier works. The interaction terms are manifestly gauge invariant because they are written in terms of gauge invariant field strengths. In this aspect it differs from BRST string field theory. BRST string field theory [30, 31, 32] also gives quadratic equations. However here (unlike in BRST string field theory), the gauge transformations of the interacting theory is the same as that of the free theory - at least up to level 
three, explicitly done in this paper. 1

Earlier work also addressed this problem from different angles. Some aspects of the finite cutoff theory has been discussed in [7, 20, 21] where it was shown that if one keeps a finite cutoff, the proper time equation for the tachyon (which in this situation is related to the RG equation), become quadratic. This is as expected both from string field theory and also from the exact renormalization group [[25]-[28]]. Some interesting aspects of the ERG have been discussed more recently in [[38]-[41]]. String field theory like equations derived from the ERG was written down in great detail in [29] and it was also shown that the S-matrix was reproduced. In [21] it was also shown that one can make precise contact with light cone string field theory by keeping a finite cutoff. In [20] it was also shown that if one wants to maintain gauge invariance while maintaining a finite cutoff one needs to include all the massive modes in the proper time equation. In this sense string field theory [31, 30, 32, 33] can be thought of as a way of keeping a finite cutoff while maintaining gauge invariance. In [23, 18] an exact ERG was written down in position space and equations of motion derived. A proposal to make it gauge invariant was given. But as mentioned above, it was not easy to write down equations in terms of space time fields, although in terms of loop variables it was particularly easy. The present version is a modification that allows one to write down equations for space time fields with relative ease. One advantage of the $\mathrm{RG}$ approach is that the background about which one perturbs does not have to be conformal. This is because the gauge invariance of the space time theory does not depend on any world sheet symmetry. This makes the construction background independent. However in this paper only the equations of motion are given. There is no attempt to construct an action.

Another approach to off shell string theory is the background independent approach of [34] and further developed in [35, 36, 37]. The connection with the RG approach is discussed in [36, 37]. There is an elegant proposal for the action. However there are problems in generalizing this proposal to general field configurations [35]. In [24] a proposal was given for the action, which in fact reduced to the above results in the near on shell limit. However the gauge invariant generalization using loop variables suffered from the same problems mentioned above (for the equations of motion). It would be interesting to see whether the techniques of this paper for gauge invariant

\footnotetext{
${ }^{1}$ It is also noteworthy that in the earlier approach of [18, the gauge transformations were affected by interactions. This happens in the form of some trace constraints that are modified by the interactions. In the present approach these constraints are not modified (at least up to level three).
} 
interacting equations of motion can be used to construct an action.

This paper is organized as follows: In Section 2 we review the derivation the ERG in position space and also the application to gauge fixed backgrounds - as one would in the "old covariant formulation" of string theory. This is a review of earlier work [23] and is included here for convenience. In section 3 we give a review of loop variables and derive the ERG using the loop variable language and we derive the gauge invariant version. This is substantially different from the approach in [23] as mentioned earlier in the introduction. Section 4 contains some explicit calculations for spin 2 and spin 3 fields. Section 5 contains some conclusions and speculations.

\section{$2 \quad$ RG in Position Space}

In this section we derive the exact $R G$ in position space. This is a repetition of Wilson's original derivation [25]. Note that usual discussions of the ERG use momentum space rather than position space. We start with point particle quantum mechanics. (This section is a review of ERG and also of some results from [23] which is reproduced here for convenience.)

\subsection{Quantum Mechanics}

Consider the Schrodinger equation

$$
i \frac{\partial \psi}{\partial t}=-\frac{\partial^{2} \psi}{\partial y^{2}}
$$

for which the Green's function is $\frac{1}{\sqrt{2 \pi\left(t_{2}-t_{1}\right)}} e^{i \frac{\left(y_{2}-y_{1}\right)^{2}}{2\left(t_{2}-t_{1}\right)}}$, and change variables $: y=x e^{\tau}, i t=e^{2 \tau}$ and $\psi^{\prime}=e^{\tau} \psi$ to get the differential equation

$$
\frac{\partial \psi^{\prime}}{\partial \tau}=\frac{\partial}{\partial x}\left(\frac{\partial}{\partial x}+x\right) \psi^{\prime}
$$

The Green's function is:

$$
G\left(x_{2}, \tau_{2} ; x_{1}, 0\right)=\frac{1}{\sqrt{2 \pi\left(1-e^{-2 \tau_{2}}\right)}} e^{-\frac{\left(x_{2}-x_{1} e^{-\tau_{2}}\right)^{2}}{2\left(1-e^{-2 \tau_{2}}\right)}}
$$

Thus as $\tau_{2} \rightarrow \infty$ it goes over to $\frac{1}{\sqrt{2 \pi}} e^{-\frac{1}{2} x_{2}^{2}}$. As $\tau_{2} \rightarrow 0$ it goes to $\delta\left(x_{1}-x_{2}\right)$.

$$
\psi\left(x_{2}, \tau_{2}\right)=\int d x_{1} G\left(x_{2}, \tau_{2} ; x_{1}, 0\right) \psi\left(x_{1}, 0\right)
$$


So $\psi\left(x_{2}, \tau_{2}\right)$ goes from being unintegrated $\psi\left(x_{1}\right)$ to completely integrated $\frac{1}{\sqrt{2 \pi}} e^{-\frac{1}{2} x_{2}^{2}} \int d x_{1} \psi\left(x_{1}\right)$. Thus consider

$$
\frac{\partial}{\partial \tau} \psi\left(x_{2}, \tau\right)=\frac{\partial}{\partial x_{2}}\left(\frac{\partial}{\partial x_{2}}+x_{2}\right) \psi\left(x_{2}, \tau\right)
$$

with initial condition $\psi(x, 0)$ Thus we can define $Z(\tau)=\int d x_{2} \psi\left(x_{2}, \tau\right)$, where $\psi$ obeys the above equation, we see that $\frac{d}{d \tau} Z=0$. When $\tau=0 \psi$ is the unintegrated $\psi(x, 0)$. At $\tau=\infty$ it is proportional to the integrated object $\int d x \psi(x, 0) . Z(\tau)$ remains the same. Thus $\tau$ measures the extant to which $Z$ is integrated.

We will now repeat this after taking the initial wave function as $e^{\frac{i}{\hbar} S[x]}$ where $x$ denotes the space-time coordinates. Then for $\tau=\infty \psi \approx \int \mathcal{D} x e^{i S[x]}$ is the integrated partition function. At $\tau=0$ it is the unintegrated $e^{i S[x]}$. $Z(\tau)$ is always the fully integrated partition function. Following [28], we shall also split the action into a kinetic term and interaction term. Thus we write $\psi=e^{-\frac{1}{2} x^{2} f(\tau)+L(x)}$ in the quantum mechanical case discussed above.

By choosing $a, b, B$ suitably $\left(b=2 a f, B=\frac{\dot{f}}{b f}\right)$ in

$$
\frac{\partial \psi}{\partial \tau}=B \frac{\partial}{\partial x}\left(a \frac{\partial}{\partial x}+b x\right) \psi(x, \tau)
$$

we get

$$
\frac{\partial L}{\partial \tau}=\frac{\dot{f}}{2 f^{2}}\left[\frac{\partial^{2} L}{\partial x^{2}}+\left(\frac{\partial L}{\partial x}\right)^{2}\right]
$$

Note that if $f=G^{-1}$ ( $G$ can be thought of as the propagator) then $\frac{\dot{f}}{f^{2}}=-\dot{G}$

\section{$2.2 \quad$ Field Theory}

We now apply this to a Euclidean field theory.

$$
\psi=e^{-\frac{1}{2} \int d z \int d z^{\prime} X(z) G^{-1}\left(z, z^{\prime}\right) X\left(z^{\prime}\right)+\int d z L\left[X(z), X^{\prime}(z)\right]}
$$

Here $X^{\prime}(z)=\partial_{z} X(z)$. In general there could be higher derivatives $X^{\prime \prime}(z), X^{\prime \prime \prime}(z) \ldots$ The equations can easily be generalized to include those cases. We apply the operator

$$
\int d z \int d z^{\prime} B\left(z, z^{\prime}\right) \frac{\delta}{\delta X\left(z^{\prime}\right)}\left[\frac{\delta}{\delta X(z)}+\int b\left(z, z^{\prime \prime}\right) X\left(z^{\prime \prime}\right)\right]
$$

to $\psi$ and require that this should be equal to $\frac{\partial \psi}{\partial \tau}$, as before,. 
We observe that

$$
\frac{\delta}{\delta X(z)} \int d u L\left[X(u), X^{\prime}(u)\right]=\int d u\left[\frac{\partial L}{\partial X(u)} \delta(u-z)+\frac{\partial L}{\partial X^{\prime}(u)} \partial_{u} \delta(u-z)\right]
$$

and also

$\frac{\delta}{\delta X\left(z^{\prime}\right)} \int d u\left[\frac{\partial L}{\partial X(u)} \delta(u-z)\right]=\int d u\left[\frac{\partial^{2} L}{\partial X(u)^{2}} \delta(u-z) \delta\left(u-z^{\prime}\right)+\frac{\partial^{2} L}{\partial X(u) \partial X^{\prime}(u)}\left[\partial_{u} \delta\left(u-z^{\prime}\right)\right] \delta(u-z)\right]$

and

$$
\begin{gathered}
\frac{\delta}{\delta X\left(z^{\prime}\right)} \int d u\left[\frac{\partial L}{\partial X^{\prime}(u)} \partial_{u} \delta(u-z)\right]=\int d u\left[\frac{\partial^{2} L}{\partial X(u) \partial X^{\prime}(u)}\left[\partial_{u} \delta(u-z)\right] \delta\left(u-z^{\prime}\right)+\right. \\
\left.\frac{\partial^{2} L}{\partial X^{\prime}(u) \partial X^{\prime}(u)}\left[\partial_{u} \delta(u-z)\right]\left[\partial_{u} \delta\left(u-z^{\prime}\right)\right]\right]
\end{gathered}
$$

Adding (2.2.9)-(2.2.10) and integrating by parts we get for the linear term:

$$
\begin{gathered}
\frac{\delta^{2}}{\delta X(z) \delta X\left(z^{\prime}\right)} \int d u L\left[X(u), X^{\prime}(u)\right]=\frac{\partial^{2} L\left[X(z), X^{\prime}(z)\right]}{\partial X(z)^{2}} \delta\left(z-z^{\prime}\right)- \\
\partial_{z}\left[\frac{\partial^{2} L\left[X(z), X^{\prime}(z)\right]}{\partial X(z) \partial X^{\prime}(z)}\right] \delta\left(z-z^{\prime}\right)+\partial_{z} \partial_{z^{\prime}}\left[\frac{\partial^{2} L\left[X(z), X^{\prime}(z)\right]}{\partial X^{\prime}(z)^{2}} \delta\left(z-z^{\prime}\right)\right]
\end{gathered}
$$

There is also a quadratic term. Again using (2.2.8) in the form

$$
\frac{\delta}{\delta X(z)} \int d u L\left[X(u), X^{\prime}(u)\right]=\left[\frac{\partial L}{\partial X(z)}-\partial_{z} \frac{\partial L}{\partial X^{\prime}(z)}\right]
$$

we get for the quadratic term

$$
\left[\frac{\partial L\left[X(z), X^{\prime}(z)\right]}{\partial X(z)}-\partial_{z} \frac{\partial L\left[X(z), X^{\prime}(z)\right]}{\partial X^{\prime}(z)}\right]\left[\frac{\partial L\left[X\left(z^{\prime}\right), X^{\prime}\left(z^{\prime}\right)\right]}{\partial X\left(z^{\prime}\right)}-\partial_{z^{\prime}} \frac{\partial L\left[X\left(z^{\prime}\right), X^{\prime}\left(z^{\prime}\right)\right]}{\partial X^{\prime}\left(z^{\prime}\right)}\right]
$$

We get the following five terms (all multiplied by $\int d z \int d z^{\prime} B\left(z, z^{\prime}\right)$ :

$$
\begin{gathered}
\left(b-G^{-1}\right)\left(z, z^{\prime}\right) \\
\left(\frac{\partial^{2} L\left[X(z), X^{\prime}(z)\right]}{\partial X(z)^{2}} \delta\left(z-z^{\prime}\right)-\partial_{z}\left[\frac{\partial^{2} L\left[X(z), X^{\prime}(z)\right]}{\partial X(z) \partial X^{\prime}(z)}\right] \delta\left(z-z^{\prime}\right)+\right. \\
\partial_{z} \partial_{z^{\prime}}\left[\frac{\partial^{2} L\left[X(z), X^{\prime}(z)\right]}{\partial X^{\prime}(z)^{2}} \delta\left(z-z^{\prime}\right)\right]
\end{gathered}
$$




$$
\begin{aligned}
& \left.\left.+\frac{\partial L\left[X(z), X^{\prime}(z)\right]}{\partial X(z)}-\partial_{z} \frac{\partial L\left[X(z), X^{\prime}(z)\right]}{\partial X^{\prime}(z)}\right]\left[\frac{\partial L\left[X\left(z^{\prime}\right), X^{\prime}\left(z^{\prime}\right)\right]}{\partial X\left(z^{\prime}\right)}-\partial_{z^{\prime}} \frac{\partial L\left[X\left(z^{\prime}\right), X^{\prime}\left(z^{\prime}\right)\right]}{\partial X^{\prime}\left(z^{\prime}\right)}\right]\right) \\
& +\frac{\partial L}{\partial X(z)}\left(-\int G^{-1}\left(z^{\prime}, z^{\prime \prime}\right) X\left(z^{\prime \prime}\right) d z^{\prime \prime}\right) \\
& +\frac{\partial L}{\partial X\left(z^{\prime}\right)}\left(\int\left(b-G^{-1}\right)\left(z, z^{\prime \prime}\right) X\left(z^{\prime \prime}\right) d z^{\prime \prime}\right) \\
& \quad-\left[\left(b-G^{-1}\right) X\right](z)\left[G^{-1} X\right]\left(z^{\prime}\right)
\end{aligned}
$$

The first term is independent of $X$ and is therefore an unimportant overall constant. If we choose $b=2 G^{-1}$, the third and fourth terms add up to zero (since $B\left(z, z^{\prime}\right)$ is symmetric under interchange of $\left.z, z^{\prime}\right)$.

Thus the second term becomes

$$
\begin{aligned}
& \int d z \int d z^{\prime} B\left(z, z^{\prime}\right)\left(\left(\frac{\partial^{2} L\left[X(z), X^{\prime}(z)\right]}{\partial X(z)^{2}} \delta\left(z-z^{\prime}\right)-\partial_{z}\left[\frac{\partial^{2} L\left[X(z), X^{\prime}(z)\right]}{\partial X(z) \partial X^{\prime}(z)}\right] \delta\left(z-z^{\prime}\right)+\right.\right. \\
& + \\
& \left.\partial_{z} \partial_{z^{\prime}}\left[\frac{\partial^{2} L\left[X(z), X^{\prime}(z)\right]}{\partial X^{\prime}(z)^{2}} \delta\left(z-z^{\prime}\right)\right]\right) \\
& \left.\left[\frac{\partial L\left[X(z), X^{\prime}(z)\right]}{\partial X(z)}-\partial_{z} \frac{\partial L\left[X(z), X^{\prime}(z)\right]}{\partial X^{\prime}(z)}\right]\left[\frac{\partial L\left[X\left(z^{\prime}\right), X^{\prime}\left(z^{\prime}\right)\right]}{\partial X\left(z^{\prime}\right)}-\partial_{z^{\prime}} \frac{\partial L\left[X\left(z^{\prime}\right), X^{\prime}\left(z^{\prime}\right)\right]}{\partial X^{\prime}\left(z^{\prime}\right)}\right]\right)
\end{aligned}
$$

and the last term becomes:

$$
-\int d z \int d z^{\prime} B\left(z, z^{\prime}\right) d z^{\prime \prime} d z^{\prime \prime \prime} G^{-1}\left(z, z^{\prime \prime}\right) X\left(z^{\prime \prime}\right) G^{-1}\left(z^{\prime}, z^{\prime \prime \prime}\right) X\left(z^{\prime \prime \prime}\right) \psi
$$

We can set (2.2.7) equal to

$$
\frac{\partial \psi}{\partial \tau}=-\frac{1}{2} \int d z \int d z^{\prime} X(z) \frac{\partial G^{-1}}{\partial \tau}\left(z, z^{\prime}\right) X\left(z^{\prime}\right) \psi+\int d z \frac{\partial L}{\partial \tau} \psi
$$

This ensures that $Z=\int \mathcal{D} X \psi$ satisfies $\frac{\partial Z}{\partial \tau}=0$. If we now set $B=$ $-\frac{1}{2} \dot{G}^{-1}\left(z, z^{\prime}\right)$ the equation for $\psi$ reduces to:

$$
\int d z \frac{\partial L}{\partial \tau}=-\int d z \int d z^{\prime} \frac{1}{2} \dot{G}\left(z, z^{\prime}\right)\left(\left(\frac{\partial^{2} L\left[X(z), X^{\prime}(z)\right]}{\partial X(z)^{2}} \delta\left(z-z^{\prime}\right)-\partial_{z}\left[\frac{\partial^{2} L\left[X(z), X^{\prime}(z)\right]}{\partial X(z) \partial X^{\prime}(z)}\right] \delta\left(z-z^{\prime}\right)+\right.\right.
$$




$$
\begin{gathered}
\left.\partial_{z} \partial_{z^{\prime}}\left[\frac{\partial^{2} L\left[X(z), X^{\prime}(z)\right]}{\partial X^{\prime}(z)^{2}} \delta\left(z-z^{\prime}\right)\right]\right) \\
\left.+\left(\left[\frac{\partial L\left[X(z), X^{\prime}(z)\right]}{\partial X(z)}-\partial_{z} \frac{\partial L\left[X(z), X^{\prime}(z)\right]}{\partial X^{\prime}(z)}\right]\left[\frac{\partial L\left[X\left(z^{\prime}\right), X^{\prime}\left(z^{\prime}\right)\right]}{\partial X\left(z^{\prime}\right)}-\partial_{z^{\prime}} \frac{\partial L\left[X\left(z^{\prime}\right), X^{\prime}\left(z^{\prime}\right)\right]}{\partial X^{\prime}\left(z^{\prime}\right)}\right]\right)\right)
\end{gathered}
$$

We can now take $\tau \approx \ln a$ and then this becomes easy to interpret as an RG equation diagrammatically [28]: the first curved bracket in the RHS which is linear in $L$ represents contractions of fields at the same point - self contractions within an operator. These can be understood as a prefactor multiplying normal ordered vertex operators. The second curved bracket represents contractions between fields at two different points - between two different operators. In terms of space-time fields, first term gives the free equations of motion and the second gives the interactions.

\subsection{ERG in the Old Covariant Formalism}

We assume that the action is

$$
\begin{aligned}
S & =\int_{0}^{R} d z L[X(z)]=\int_{0}^{R} d z \int d k\left[\frac{\phi(k)}{a} e^{i k X(z)}+A_{\mu}(k) \partial_{z} X^{\mu} e^{i k X(z)}\right. \\
& \left.+\frac{1}{2} a S_{2}(k)^{\mu} \partial_{z}^{2} X^{\mu} e^{i k X(z)}+a S^{\mu \nu}(k) \partial X^{\mu} \partial X^{\nu} e^{i k X(z)}+\ldots\right]
\end{aligned}
$$

$a$ is a short distance cutoff. In order to implement the ERG we also need a specific form for $\dot{G}\left(z, z^{\prime}\right)=\dot{G}\left(z-z^{\prime}\right)$. We need $\dot{G}(u)$ to be short ranged, otherwise the dimensionless ratio $\frac{R}{a}$ will enter in the equations. It is also a good idea to have analyticity so that one can perform Taylor expansions, which are required when we do OPE's. We will use the cutoff Green's function:

$$
G(u)=\int \frac{d^{2} k}{(2 \pi)^{2}} \frac{e^{i k u} e^{-a^{2} k^{2}}}{k^{2}}
$$

This has a cutoff at short distances of $O(a)$ and at long distances reduces to the usual propagator. We now apply the ERG (2.2.16) to the action S (2.3.17).

The LHS gives

$$
\int d z \int d k\left[\frac{\beta_{\phi(k)}-\phi(k)}{a} e^{i k_{0} Y}+\beta_{A^{\mu}(k)} \partial_{z} X^{\mu}(z) e^{i k_{0} Y}+\ldots\right]
$$

where $\beta_{g} \equiv \dot{g}$. The first term of the RHS gives

$$
\int d z \int d k \frac{1}{2}\left(-k^{2}\right) \frac{e^{i k_{0} Y}}{a} \phi(k)
$$


The second term gives

$$
\int d k_{1} \int d k_{2} \frac{\phi\left(k_{1}\right) \phi\left(k_{2}\right)}{a^{2}}\left(\frac{-k_{1} \cdot k_{2}}{2}\right) \int_{-R}^{+R} d u \dot{G}(u) e^{i k_{1} \cdot X(z)} e^{i k_{2} \cdot X(z+u)}
$$

One can do an OPE for the product of exponentials to get 2

$$
e^{i\left(k_{1}+k_{2}\right) X(z)+i k_{1}\left[u \partial_{z} X+\frac{u^{2}}{2} \partial^{2} X+\ldots\right]}
$$

This gives

$$
\begin{gathered}
e^{i\left(k_{1}+k_{2}\right) X(z)} \int_{-R}^{+R} d u \dot{G}(u)+i k_{1} \partial X e^{i\left(k_{1}+k_{2}\right) X(z)} \int_{-R}^{+R} d u u \dot{G}(u) \\
+i k_{1} \frac{\partial^{2} X}{2} e^{i\left(k_{1}+k_{2}\right) X(z)} \int_{-R}^{+R} d u u^{2} \dot{G}(u)+\frac{i k_{\mu} i k_{\nu}}{2} \partial X^{\mu} \partial X^{\nu} e^{i\left(k_{1}+k_{2}\right) X(z)} \int_{-R}^{+R} d u u^{2} \dot{G}(u)
\end{gathered}
$$

It is easy to see that the first term of the OPE contributes to the tachyon equation:

$\beta_{\phi(k)}-\phi(k)=\phi(k)\left(\frac{-k^{2}}{2}\right)-\frac{1}{2} \int d k_{1} \phi\left(k_{1}\right) \phi\left(k-k_{1}\right) \frac{k_{1} \cdot\left(k-k_{1}\right)}{2 a} \int_{-R}^{+R} d u \dot{G}(u)$

Similarly the second term of the OPE gives the photon equation:

$$
\beta_{A^{\mu}(k)}=\int d k_{1} \frac{\phi\left(k_{1}\right) \phi\left(k-k_{1}\right)}{a^{2}}\left(\frac{-k_{1}\left(. k-k_{1}\right)}{2}\right) i k_{1}^{\mu} \int_{-R}^{+R} d u u \dot{G}(u)
$$

This gives the the tachyon contribution to the tachyon and photon equations of motion (EOM). More precisely these are contributions to the beta functions. The proportionality factor relating the beta function and the EOM is the Zamolodchikov metric. Note that there is a dependence on $R / a$ in the coefficients, but from the form of the cutoff chosen it is easy to see that there are always factors of $e^{-\frac{R^{2}}{a^{2}}}$ accompanying the cutoff dependent terms and one can safely take the limit $R \rightarrow \infty$ when this dependence disappears. The equations then become independent of $a$ - even though $a$ is finite. Thus the finite $a$ equations have the same form as the continuum equations. This is what is envisaged in the "improved" actions [43] or "perfect" actions [44]. Furthermore if the fields are tuned to the fixed point values, such that the beta function vanishes, then we have scale invariance at finite cutoff.

\footnotetext{
${ }^{2}$ The OPE in terms of normal ordered is discussed in the Appendix. We will use that to get the contribution of higher levels to lower level field equations. Here we do not normal order.
} 
One can also include the contribution due to the photon field:

$$
\begin{gathered}
\frac{\delta}{\delta X^{\mu}(z)} \int d z^{\prime \prime} \int d k A_{\nu}(k) \partial_{z^{\prime \prime}} X^{\nu}\left(z^{\prime \prime}\right) e^{i k X\left(z^{\prime \prime}\right)} \\
=\int d z^{\prime \prime} \int d k A_{\nu}(k)\left[\delta^{\mu \nu} \partial_{z^{\prime \prime}} \delta\left(z-z^{\prime \prime}\right) e^{i k X\left(z^{\prime \prime}\right)}+\partial_{z^{\prime \prime}} X^{\nu}\left(z^{\prime \prime}\right) i k^{\mu} \delta\left(z-z^{\prime \prime}\right) e^{i k_{0} Y}\right] \\
=\int d z^{\prime \prime} \int d k A_{\nu}(k)\left[-\delta^{\mu \nu} \delta\left(z-z^{\prime \prime}\right) i k^{\rho} \partial_{z^{\prime \prime}} X^{\rho} e^{i k X\left(z^{\prime \prime}\right)}+\partial_{z^{\prime \prime}} X^{\nu}\left(z^{\prime \prime}\right) i k^{\mu} \delta\left(z-z^{\prime \prime}\right) e^{i k_{0} Y}\right. \\
=\int d z^{\prime \prime} \int d k\left[-A^{\mu}(k) i k^{\nu}+i k^{\mu} A^{\nu}\right] \partial_{z^{\prime \prime}} X^{\nu}\left(z^{\prime \prime}\right) \delta\left(z-z^{\prime \prime}\right) e^{i k_{0} Y} \\
\frac{\delta^{2}}{\delta X^{\mu}\left(z^{\prime}\right) X^{\mu}(z)} \int d z L=\int d z^{\prime \prime} \int d k \delta\left(z-z^{\prime \prime}\right)[\partial_{z^{\prime \prime}} \delta\left(z^{\prime \prime}-z^{\prime}\right) \underbrace{\delta^{\nu \mu}\left[-i k^{\nu} A^{\mu}+i A^{\nu} k^{\mu}\right]}_{=0} e^{i k_{0} Y} \\
+\partial_{z^{\prime \prime}} X^{\nu}\left[-i k^{\nu} A^{\mu}+i k^{\mu} A^{\nu}\right] i k^{\mu} \delta\left(z^{\prime \prime}-z^{\prime}\right)
\end{gathered}
$$

The second term $\frac{\partial L}{\partial X^{\mu}(z)} \frac{\partial L}{\partial X^{\mu}\left(z^{\prime}\right)}$ becomes

$$
\begin{gathered}
\int d z^{\prime \prime} \int d k\left[-A^{\mu}(k) i k^{\nu}+i k^{\mu} A^{\nu}\right] \partial_{z^{\prime \prime}} X^{\nu}\left(z^{\prime \prime}\right) \delta\left(z-z^{\prime \prime}\right) e^{i k X\left(z^{\prime \prime}\right)} \\
\int d z^{\prime \prime \prime} \int d k\left[-A^{\mu}(k) i k^{\nu}+i k^{\mu} A^{\nu}\right] \partial_{z^{\prime \prime \prime}} X^{\nu}\left(z^{\prime \prime \prime}\right) \delta\left(z^{\prime}-z^{\prime \prime \prime}\right) e^{i k X\left(z^{\prime \prime \prime}\right)}
\end{gathered}
$$

Thus putting everything together we get

$$
\begin{aligned}
& \int d z \int d z^{\prime} \dot{G}\left(z-z^{\prime}\right)\left\{\int d k\left[-A^{\mu}(k) i k^{\nu}+i k^{\mu} A^{\nu}\right] i k^{\mu} \partial_{z^{\prime}} X^{\nu}\left(z^{\prime}\right) \delta\left(z-z^{\prime}\right) e^{i k_{0} Y}\right. \\
& \left.+\int d k \int d k^{\prime}\left[-i k^{[\rho} A^{\mu]}\right]\left[-i k^{\prime[\sigma} A^{\mu]}\right] \partial_{z} X^{\rho}(z) e^{i k_{0} Y} \partial_{z^{\prime}} X^{\sigma}\left(z^{\prime}\right) e^{i k^{\prime} X\left(z^{\prime}\right)}\right\}
\end{aligned}
$$

The first term is the usual Maxwell equation of motion. If we assume analyticity of $\left.\dot{G}\left(z-z^{\prime}\right)\right)$ we can perform an OPE in the second term and re-express as a sum of vertex operators for the various modes, just as in the case of the tachyon, above.

The gauge invariance follows because of the integral over $z, z^{\prime}$ which allows integration by parts. For the same reason, we have seen in loop variable calculations that if we do not introduce additional coordinates for higher gauge invariances, one need not expect full gauge invariance in the EOM for higher (massive) modes. The gauge invariance due to $L_{-1}$ is present as the freedom to add total divergences in $z$. For the higher gauge invariances due to $L_{-2}, L_{-3} \ldots$ we need to be able to add total derivatives in some additional variables. This will be reviewed in the next section. 


\section{Loop Variables and the ERG}

\subsection{Loop Variables}

Loop variables are useful when one wants to have a completely general background i.e. when all the massive modes are turned on as background. This is the domain of string field theory and thus one is working with the full string field $\Phi[X(s)]$. Thus for instance,

$$
\Phi[X(s)]=\int[d k(s)] e^{i \int_{c} d s k(s) X(s)} \Phi[k(s)]
$$

In the limit $a \rightarrow 0$ we have a collection of vertex operators, all at the same point $z$. We have the following Taylor expansion:

$$
X(z+a s)=X(z)+a s \partial_{z} X(z)+\frac{1}{2 !} a^{2} s^{2} \partial_{z}^{2} X(z)+\ldots
$$

We also assume that $k(s)$ can be expanded in a power series in $1 / s$. Thus

$$
k(s)=k_{0}+\frac{k_{1}}{s}+\frac{k_{2}}{s^{2}}+\ldots
$$

Instead of (3.1.26), for later convenience we use the following definition of the loop variable:

$$
e^{i k_{0} X(z)+i \int_{c} d s k(s) \partial_{z} X(z+a s)}
$$

When we expand the exponential in a power series we get the following terms:

$e^{i k_{0} X(z)+i \int_{c} d s k(s) \partial_{z} X(z+a s)}=e^{i k_{0} X(z)}\left[1+i k_{1}^{\mu} \partial_{z} X^{\mu}-\frac{1}{2} k_{1}^{\mu} k_{1}^{\nu} \partial_{z} X^{\mu} \partial_{z} X^{\nu}+k_{2}^{\mu} \partial^{2} X^{\mu}+\ldots\right]$

These are precisely the terms one writes down when one considers an open string in a general background, except that they are written in terms of loop variable momenta rather than space time fields. The connection becomes precise when we define space time fields in terms of loop variables.

The space time fields are defined by relations of the form:

$$
\begin{gathered}
\langle 1\rangle=\phi \\
\left\langle k_{1}^{\mu}\right\rangle=A^{\mu} \\
\left\langle k_{2}^{\mu}\right\rangle=S_{2}^{\mu}
\end{gathered}
$$




$$
\left\langle k_{1}^{\mu} k_{1}^{\nu}\right\rangle=S_{11}^{\mu \nu}
$$

where the $\langle.$.$\rangle indicates an integration over some string functional of the$ $k_{n}, 3$ Thus for instance,

$$
\left\langle k_{1}^{\mu}\right\rangle \equiv \int \underbrace{\mathcal{D} k(s)}_{\left[d k_{1} d k_{2} \ldots d k_{n} \ldots\right]} k_{1}^{\mu} \Psi\left[k_{0}, k_{1}, k_{2}, \ldots k_{n} . . ; \phi, A^{\mu}, S^{\mu \nu} \ldots\right]=A^{\mu}\left(k_{0}\right)
$$

We have not done the integral over $k_{0}$, the usual space time momentum. Thus our fields are in momentum space. This is only for convenience. One could integrate over $k_{0}$ and include $e^{i k_{0} \cdot X(z)}$ in $\Psi$ if one wanted.

Note that the loop variable can also be written as

$$
e^{i \sum_{n \geq 0} k_{n}(t) \tilde{Y}_{n}(t)}
$$

where

$$
\tilde{Y}_{n} \equiv \frac{1}{(n-1) !} \partial_{z}^{n} X(z), n>0 ; \quad \tilde{Y}_{0} \equiv X(z)
$$

\subsection{Gauge Invariance}

We have seen that gauge invariance follows from the freedom to add derivatives. Thus if one is to retain all derivative terms one needs a local RG where the cutoff $a$ depends on $z$. This is illustrated below.

One can impose scale invariance by requiring that cutoff dependence vanish in expectation values $\left\langle O_{i}\right\rangle$. This is equivalent to evaluating the effects of normal ordering.

Thus for the tachyon, $\frac{1}{a} e^{i k \cdot X}=e^{\left(\frac{k^{2}}{2 \pi}-1\right) \ln a}: e^{i k \cdot X}:$. Here :..: denotes normal ordering, so that $\langle: O:\rangle \equiv 0$ for all operators, except for the exponential : $\left\langle: e^{i k_{0} Y}:\right\rangle \equiv 1$. $\frac{d}{d l n a}=0$ gives the equation of motion of the tachyon. Let us do this for the vector. But first we replace $a$ by $a e^{\sigma(z)}-$ this makes it local. $\sigma(z)$ can be thoought of as the Liouville mode.

$$
A^{\mu}(k) \partial_{z} X^{\mu} e^{i k . X}=-i k \cdot A(k) \frac{\partial_{z} \sigma}{2 \pi}: e^{i k \cdot X}: e^{\frac{k^{2}}{2 \pi} \sigma}+A^{\mu}(k): \partial_{z} X^{\mu} e^{i k . X}: e^{\frac{k^{2}}{2 \pi} \sigma}
$$

Now we vary w.r.t $\sigma$, and integrate by parts, and then set $\sigma=0$ to find,

$$
\left(-k \cdot A(k) k^{\mu}+k^{2} A^{\mu}(k)\right): \partial_{z} X^{\mu} e^{i k \cdot X}:
$$

\footnotetext{
${ }^{3}$ The brackets \langle\rangle are also being used to denote the usual field theoretic correlations. It should be clear from the context which is intended.
} 
This is Maxwell's equation. The $\partial_{z} \sigma$ piece is crucial. The two physical state conditions $L_{0}=0$ and $L_{1}=0$ are combined into one equation. The gauge invariance of the equation ensures that this one equation is equivalent to both constraints. This shows the role of local scale invariance.

Suppose attempt to do the same thing for the massive modes of the form $S_{2} \partial_{z}^{2} X e^{i k . X}$. One expects terms, for instance, of the form $k . S(k) \partial_{z}^{2} \sigma: e^{i k . X}:$ On varying w.r.t $\sigma$, the $z$ derivative acts twice on $e^{i k . X}$ and, we get terms of the form $k . S(k) k^{\mu} k^{\nu}: \partial_{z} X^{\mu} \partial_{z} X^{\nu} e^{i k . X}:$. The resulting equation is cubic in derivatives and is not acceptable as an equation of motion. It doesn't get better at higher levels [15.

So following [15] we introduce additional variables $x_{n}, n>0$ that have the property that $\frac{\partial}{\partial x_{n}} \approx \partial_{z}^{n}$. On integrating by parts (and acting on $e^{i k_{0} Y}$ ), instead of getting $n$ powers of momenta, we get one.

What could be the origin of these extra variables? They can be thought of as parametrizing diffeomorphisms of the loop variable. As it stands the loop variable is not diffeomorphism invariant but we can make it so by introducing an "einbein" along the loop. The modes of this einbein then provide the extra variables $x_{n}$.

Let us consider the following loop variable:

$$
e^{i \int_{c} \alpha(t) k(t) \partial_{z} X(z+t) d t+i k_{0} X}
$$

$\alpha(s)$ is an einbein. Let us assume the following Laurent expansion:

$$
\alpha(s)=1+\frac{\alpha_{1}}{s}+\frac{\alpha_{2}}{s^{2}}+\frac{\alpha_{3}}{s^{3}}+\ldots
$$

Let us define

$$
\begin{aligned}
Y & =X+\alpha_{1} \partial_{z} X+\alpha_{2} \partial_{z}^{2} X+\alpha_{3} \frac{\partial_{z}^{3} X}{2}+\ldots+\frac{\alpha_{n} \partial_{z}^{n} X}{(n-1) !}+\ldots \\
& =X+\sum_{n>0} \alpha_{n} \tilde{Y}_{n} \\
Y_{1} & =\partial_{z} X+\alpha_{1} \partial_{z}^{2} X+\alpha_{2} \frac{\partial_{z}^{3} X}{2}+\ldots+\frac{\alpha_{n-1} \partial_{z}^{n} X}{(n-1) !}+\ldots \\
\ldots & \cdots \\
Y_{m} & =\frac{\partial_{z}^{m} X}{(m-1) !}+\sum_{n>m} \frac{\alpha_{n-m} \partial_{z}^{n} X}{(n-1) !}
\end{aligned}
$$

Define $\alpha_{0}=1$ so that the $>$ signs in the summations above can be replaced by $\geq$. 
Thus

$$
e^{i \int_{c} \alpha(t) k(t) \partial_{z} X(z+t) d t+i k_{0} X}=e^{i \sum_{n} k_{n} Y_{n}}
$$

where $Y_{0}=Y$.

Introduce $x_{n}$ by the following:

$$
\alpha(s)=\sum_{n \geq 0} \alpha_{n} s^{-n}=e^{\sum_{m \geq 0} s^{-m} x_{m}}
$$

Thus

$$
\begin{aligned}
\alpha_{1} & =x_{1} \\
\alpha_{2} & =\frac{x_{1}^{2}}{2}+x_{2} \\
\alpha_{3} & =\frac{x_{1}^{3}}{3 !}+x_{1} x_{2}+x_{3}
\end{aligned}
$$

They satisfy,

$$
\frac{\partial \alpha_{n}}{\partial x_{m}}=\alpha_{n-m}, \quad n \geq m
$$

and thus

$$
Y_{n}=\frac{\partial Y}{\partial x_{n}}
$$

Let us define $\Sigma=\langle Y(z) Y(z)\rangle$. This is a generalization of $\sigma$ to include the $x_{n}$ dependence, just as $Y$ is a generalization of $X$. It is equal to the previous $\sigma$ when $\alpha(s)=1$.

Thus the coincident two point functions become:

$$
\begin{aligned}
\langle Y Y\rangle & =\Sigma \\
\left\langle Y_{n} Y\right\rangle & =\frac{1}{2} \frac{\partial \Sigma}{\partial x_{n}} \\
\left\langle Y_{n} Y_{m}\right\rangle & =\frac{1}{2}\left(\frac{\partial^{2} \Sigma}{\partial x_{n} \partial x_{m}}-\frac{\partial \Sigma}{\partial x_{n+m}}\right)
\end{aligned}
$$

We normal order vertex operators as before to get:

$$
\begin{aligned}
e^{i \int_{c} \alpha(t) k(t) \partial_{z} X(z+t) d t+i k_{0} X}= & e^{i \sum_{n} k_{n} Y_{n}} \\
= & \exp \left\{k_{0}^{2} \Sigma+\sum_{n>0} k_{n} \cdot k_{0} \frac{\partial \Sigma}{\partial x_{n}}+\right. \\
& \left.\sum_{n, m>0} k_{n} \cdot k_{m} \frac{1}{2}\left(\frac{\partial^{2} \Sigma}{\partial x_{n} \partial x_{m}}-\frac{\partial \Sigma}{\partial x_{n+m}}\right)\right\} \\
& : e^{i \sum_{n} k_{n} Y_{n}}:
\end{aligned}
$$


Let us set $\frac{\delta}{\delta \Sigma}$ to zero (and also set $\Sigma=0$ ). As an illustration:

$\frac{\delta}{\delta \Sigma}\left[k_{n} \cdot k_{m} \frac{1}{2}\left(\frac{\partial^{2} \Sigma}{\partial x_{n} \partial x_{m}}-\frac{\partial \Sigma}{\partial x_{n+m}}\right)\right]: e^{i k_{0} . Y}:=:\left(\frac{1}{2} i k_{0}^{\mu} i k_{0}^{\nu} Y_{n}^{\mu} Y_{m}^{\nu}+i k_{0}^{\mu} Y_{n+m}^{\mu}\right) e^{i k_{0} \cdot Y}:$

If we now collect all the coefficients of a particular vertex operator, say $: Y_{n}^{\mu} e^{i k_{0} . Y}$ :, we get the free equation of motion. We can easily see that they never contain more than two space-time derivatives.

We can also understand gauge invariance as follows. Having introduced an einbein we have to integrate over all possible einbein fields, with a suitable measure $\mathcal{D} \alpha(s)$. It is this integration which allows us to integrate by parts on the $x_{n}$.

Consider the following transformation:

$$
k(s) \rightarrow \lambda(s) k(s)
$$

Clearly this is equivalent to $\alpha(s) \rightarrow \lambda(s) \alpha(s)$. But this is just a change of an integration variable. Assuming the measure is invariant this does nothing to the integral. We choose $\mathcal{D} \alpha(s)$ to be $\prod_{n} d x_{n}$ and set $\lambda(s)=e^{\sum_{m} y_{m} s^{-m}}$. Then the gauge transformation (3.2.43) is just a translation, $x_{n} \rightarrow x_{n}+y_{n}$ and leaves the measure invariant. Thus we conclude that (3.2.43) is a gauge transformation. 4

We expand $\lambda(s)$ in inverse powers of $s$

$$
\lambda(s)=\sum_{n} \lambda_{n} s^{-n}
$$

and write (3.2.43) as

$$
k_{n} \rightarrow \sum_{m=0}^{n} \lambda_{m} k_{n-m}
$$

We set $\lambda_{0}=1$.

We can interpret these equations in terms of space-time fields if we use (3.1.29), suitably extended to include $\lambda$. Thus we must assume that the string wave-functional is also a functional of $\lambda(s)$. Thus set

$$
\begin{aligned}
\left\langle\lambda_{1}\right\rangle & =\Lambda_{1}\left(k_{0}\right) \\
\left\langle\lambda_{1} k_{1}^{\mu}\right\rangle & =\Lambda_{11}^{\mu}\left(k_{0}\right) \\
\left\langle\lambda_{2}\right\rangle & =\Lambda_{2}\left(k_{0}\right)
\end{aligned}
$$

\footnotetext{
${ }^{4}\left[L_{-n}, Y_{m}\right]=m Y_{m+n}=m \frac{\partial}{\partial x_{n}} Y_{m}$. This gives the connection between the symmetry (Diff $\left.\left(S^{1}\right)\right)$ transformation in string theory and these gauge transformations.
} 
The gauge transformations (3.2.44) in terms of space time fields are given by evaluating $\langle.$.$\rangle :$

$$
\begin{aligned}
A^{\mu}\left(k_{0}\right) & \rightarrow A^{\mu}(k o)+k_{0}^{\mu} \Lambda_{1}\left(k_{0}\right) \\
S_{2}^{\mu}\left(k_{0}\right) & \rightarrow S_{2}^{\mu}(k o)+k_{0}^{\mu} \Lambda_{2}\left(k_{0}\right)+\Lambda_{11}^{\mu} \\
S_{11}^{\mu \nu} & \rightarrow S_{11}^{\mu \nu}+k_{0}^{(\mu} \Lambda_{11}^{\nu)}
\end{aligned}
$$

These are the canonical gauge transformations for a spin two field. 5

Now the gauge transformation parameters of higher spin fields obey a certain tracelessness condition [16, 17]. We will see this below also.

When one actually performs the gauge transformation it changes the normal ordered loop variable by a total derivative in $x_{n}$ which doesn't affect the equation of motion. Thus the gauge variation of the loop variable is a term of the form $\frac{d}{d x_{n}}[A(\Sigma) B]$, where $B$ doesn't depend on $\Sigma$. The coefficient of $\delta \Sigma$ is obtained as

$$
\begin{gathered}
\int \delta\left(\frac{d}{d x_{n}}[A(\Sigma) B]\right)=\int\left(\frac{d}{d x_{n}}\left(\frac{\delta A}{\delta \Sigma} \delta \Sigma\right) B+\frac{\delta A}{\delta \Sigma} \delta \Sigma \frac{d B}{d x_{n}}\right) \\
=\int\left[-\frac{\delta A}{\delta \Sigma} \frac{d B}{d x_{n}}+\frac{\delta A}{\delta \Sigma} \frac{d B}{d x_{n}}\right] \delta \Sigma=0
\end{gathered}
$$

Note that we have integrated by parts.

Actually one finds on explicit calculation that the variation is a total derivative only if we use some identities that constrain the form of $\Sigma$. However we would like to leave $\Sigma$ unconstrained when we vary. Thus constraints have to be imposed elsewhere. It turns out that the terms that have to be put to zero are all of the form

$$
\lambda_{n} k_{m} \cdot k_{p} \cdots
$$

where $\ldots$ refers to any other factors of $k_{m}$ [15. Thus all traces of gauge parameters have to be set to zero.

In [15] spin-2 and spin-3 are explicitly worked out.

The gauge transformation (3.2.43) is a scale transformation in spacetime. It is local along the loop. This is suggestive of a space time renormalization group interpretation of the symmetry group of string theory as speculated in [15]. This speculation was the motivation for this approach.

\footnotetext{
${ }^{5}$ Later a dimensional reduction will be done that make the fields massive.
} 


\subsection{Dimensional Reduction}

The equations that one obtains following the above steps give massless equations of motion.

In Section 2 the mass, being the dimension of the operator, was obtained from the canonical dimension of operators. This is just the number of derivatives. In the $\mathrm{RG}$ we introduce powers of $a e^{i \sigma}$ to make the derivatives dimensionless and so when we count powers of $a$ we get the canonical dimension. In the new scheme we need to introduce it in a way consistent with the gauge invariance of the massless theory. We simply do a KaluzaKlein reduction and thus we must let the momentum $k_{0}^{\mu}$ be a 27 -dimensional vector rather than a 26-dimensional one. We will let $k_{0}^{26} \equiv q_{0}$ stand for the mass as in Kaluza-Klein theories but we will assume that $q_{0}^{2}$ is a multiple of $\frac{1}{R^{2}}$ rather than letting $k_{0}$ be multiples of $\frac{1}{R}$. The extra dimension brings an infinite set of auxiliary fields with it. This fortunately is just what we need in string theory as shown in [30]. There it was shown that one can get all the necessary auxiliary fields from the bosonized ghost - except that the the first oscillator mode was set to zero.

We thus set $q_{0}$ to $\sqrt{(P-1)}$, where $P$ is the engineering dimension of the vertex operator. Thus for the tachyon $P=0$, for the vector $P=1$ etc. But in our case the first mode $q_{1}$ will not be set to zero identically because that would violate gauge invariance. We will impose relations consistent with gauge invariance that allow us to get rid of $q_{1}$. These are given below along with definitions of space-time fields in terms of loop variables:

Level 2:

$$
\begin{gathered}
\left\langle q_{1}\right\rangle=0 . \\
\left\langle q_{1} q_{1}\right\rangle=\left\langle q_{2} q_{0}\right\rangle=S_{2} q_{0} ;\left\langle\lambda_{1} q_{1}\right\rangle=\left\langle\lambda_{2} q_{0}\right\rangle=\Lambda_{2} q_{0} . \\
\left\langle q_{1} k_{1}^{\mu}\right\rangle=\left\langle k_{2}^{\mu} q_{0}\right\rangle=S_{2}^{\mu} q_{0} .
\end{gathered}
$$

This implies

$$
q_{2} \rightarrow q_{2}+2 \lambda_{2} q_{0}
$$

Level 3:

$$
\begin{gathered}
\left\langle q_{1} k_{1}^{\mu} k_{1}^{\nu}\right\rangle=\frac{1}{2}\left\langle k_{2}^{(\mu} k_{1}^{\nu)} q_{0}\right\rangle=\frac{1}{2} S_{21}^{(\mu \nu)} q_{0} \\
\left\langle q_{1} q_{1} k_{1}^{\mu}\right\rangle=\left\langle k_{3}^{\mu} q_{0}^{2}\right\rangle=S_{3}^{\mu} q_{0}^{2} \\
\left\langle q_{1} k_{2}^{\mu}\right\rangle=\left\langle 2 k_{3}^{\mu} q_{0}-q_{2} k_{1}^{\mu}\right\rangle=2 S_{3}^{\mu} q_{0}-S_{12}^{\mu} \\
\left\langle q_{1} q_{2}\right\rangle=\left\langle q_{3} q_{0}\right\rangle=S_{3} q_{0}
\end{gathered}
$$




$$
\begin{gathered}
\left\langle q_{1}^{3}\right\rangle=\left\langle q_{3} q_{0}^{2}\right\rangle \\
\left\langle\lambda_{1} q_{1} k_{1}^{\mu}\right\rangle=\left\langle\frac{1}{2} \lambda_{2} k_{1}^{\mu} q_{0}+\frac{1}{2} \lambda_{1} k_{2}^{\mu} q_{0}\right\rangle=\left(\frac{1}{2} \Lambda_{12}^{\mu}+\frac{1}{2} \Lambda_{21}^{\mu}\right) q_{0} \\
\left\langle\lambda_{2} q_{1}\right\rangle=\left\langle 2 \lambda_{3} q_{0}-\lambda_{1} q_{2}\right\rangle=2 \Lambda_{3} q_{0}-\Lambda_{21} \\
\left\langle\lambda_{1} q_{1} q_{1}\right\rangle=\left\langle\lambda_{3} q_{0}^{2}\right\rangle=\Lambda_{3} q_{0}^{2}
\end{gathered}
$$

The gauge transformations for fields involving $q_{2}, q_{3}$ are modified to:

$$
\delta\left(q_{2} k_{1}^{\mu}\right)=\left(\frac{3}{2} \lambda_{2} k_{1}^{\mu}+\frac{1}{2} \lambda_{1} k_{2}^{\mu}\right) q_{0}+\lambda_{1} q_{2} k_{0}^{\mu} \quad, \quad \delta q_{3}=3 \lambda_{3} q_{0}
$$

Note that correspondence with spin theory requires that $q_{0}^{2}=1$ for the level2 field and $q_{0}^{2}=2$ for the level-3 fields. Relations of this type enable us to get rid of $q_{1}$ completely. The form of the relations is such as to maintain gauge invariance.

We summarize the results for the gauge transformations of the massive spin-2 and spin 3 fields field:

\section{Level 2}

$$
\begin{gathered}
\delta S_{11}^{\mu \nu}=k_{0}^{(\mu} \Lambda_{11}^{\nu)} \\
\delta S_{2}^{\mu}=\Lambda_{11}^{\mu}+k_{0}^{\mu} \Lambda_{2} \\
\delta S_{2}=2 \Lambda_{2} q_{0}
\end{gathered}
$$

These are in the "standard" form, where the extra auxiliary fields $S_{2}$ and $S_{2}^{\mu}$ can be set to zero to recover the Pauli-Fierz equations for massive spin-2 fields. Further details can be found in [15] and references therein.

\section{Level 3}

The corresponding relations for spin 3 are as follows:

$$
\begin{gathered}
\delta S_{111}^{\mu \nu \rho}=k_{0}^{(\mu} \Lambda_{111}^{\nu \rho)} \\
\delta S_{21}^{\mu \nu}=\Lambda_{111}^{\mu \nu}+\frac{1}{2} k_{0}^{(\mu}\left(\Lambda_{12}+\Lambda_{21}\right)^{\nu)}+\frac{1}{2} k_{0}^{[\mu}\left(\Lambda_{12}-\Lambda_{21}\right)^{\nu]}
\end{gathered}
$$

If we separate the symmetric and antisymmetric parts, $S_{21}^{\mu \nu}=S^{\mu \nu}+A^{\mu \nu}$, and $\Lambda_{S}^{\mu}=\frac{1}{2}\left(\Lambda_{12}+\Lambda_{21}\right)^{\mu}$ and $\Lambda_{A}^{\mu}=\frac{1}{2}\left(\Lambda_{12}-\Lambda_{21}\right)^{\mu}$, then

$$
\begin{gathered}
\delta S^{\mu \nu}=\Lambda_{111}^{\mu \nu}+k_{0}^{(\mu} \Lambda_{S}^{\nu)} \quad ; \quad \delta A^{\mu \nu}=k_{0}^{[\mu} \Lambda_{A}^{\nu]} \\
\delta S_{3}^{\mu}=\Lambda_{21}^{\mu}+\Lambda_{12}^{\mu}+k_{0}^{\mu} \Lambda_{3}=2 \Lambda_{S}^{\mu}+k_{0}^{\mu} \Lambda_{3}
\end{gathered}
$$

$S_{3}^{\mu}$ is naturally associated with the symmetric tensor $S^{\mu \nu}$.

$$
\delta S_{12}^{\mu}=\frac{3}{2} \Lambda_{12}^{\mu} q_{0}+\frac{1}{2} \Lambda_{21}^{\mu} q_{0}+k_{0}^{\mu} \Lambda_{21} q_{0}
$$


The combination $S_{3}^{\mu} q_{0}-S_{12}^{\mu}$ undergoes the transformation

$$
\delta\left(S_{3}^{\mu} q_{0}-S_{12}^{\mu}\right)=\Lambda_{A}^{\mu} q_{0}+k_{0}^{\mu}\left(\Lambda_{21}^{\mu}-\Lambda_{3}^{\mu}\right)
$$

and is thus naturally associated with the antisymmetric tensor $A^{\mu \nu}$. Finally,

$$
\delta S_{3}=3 \Lambda_{3} q_{0}
$$

\subsection{ERG and Loop Variables}

We have already seen that what we refer to as the loop variable, integrated over $z$ i.e.

$$
\int d z \mathcal{D} \alpha(t) e^{i \int_{c} \alpha(t) k(t) \partial_{z} X(z+t) d t+i k_{0} X}
$$

is actually the interacting part of the action expressed in terms of the loop variable momenta $k_{n}$ :

$$
=\int \underbrace{\left[d z d x_{1} d x_{2} \ldots d x_{n} \ldots\right]}_{[d z]} e^{i \sum_{n} k_{n} Y_{n}}=\int[d z] L\left[Y\left(z, x_{n}\right), \frac{\partial Y}{\partial x_{1}}, \frac{\partial Y}{\partial x_{2}}, \ldots, \frac{\partial Y}{\partial x_{n}}\right]
$$

Thus the variable $z$ now stands for $\left(z, x_{1}, x_{2}, \ldots, x_{n}, \ldots\right)$. Furthermore when we have two points, $z, z^{\prime}$, they will denote the sets of variables:

$$
\left(z_{A}, x_{1 A}, x_{2 A}, \ldots, x_{n A}, \ldots\right),\left(z_{B}, x_{1 B}, x_{2 B}, \ldots, x_{n A}, \ldots\right)
$$

The integrals $\int d z$ in the ERG will be replaced by $\int \ldots \int d z d x_{1 A} d x_{2 A} . . d x_{n A} \ldots$ Thus we will be allowed to integrate by parts on the $x_{n}$ 's exactly as in the case of the free string described above. We will see that the linear terms in the ERG equation reproduce the free string equation and the quadratic term describes the interactions. The interactions will turn out to be not fully gauge invariant. The full gauge invariance requires a further modification described later below.

\subsection{Free Equations}

The free equations are obtained from the terms that are linear in $L$ in (2.2.16) . We have to extend the meaning of $X^{\prime}(z)$ to $Y_{n}=\frac{\partial Y}{x_{n}}$ for all $n$. Thus $\frac{\partial^{2} L}{\partial X^{2}}$ becomes $\frac{\partial^{2} L}{\partial Y^{2}}$ in our new notation. Noting that in (2.2.16), $G\left(z, z^{\prime}\right)=$ $\left\langle X(z) X\left(z^{\prime}\right)\right\rangle$, we see that in the gauge invariant version, $\delta\left(z-z^{\prime}\right)$ in the first term is actually $\delta\left(z-z^{\prime}\right) \prod_{n} \delta\left(x_{n}-x_{n}^{\prime}\right)$ and so $G(z, z)=\langle Y(z) Y(z)\rangle=\Sigma$ in the notation of Section 3. 
The loop variable is

$$
e^{i\left(k_{0}^{\mu} Y^{\mu}+k_{1}^{\mu} Y_{1}^{\mu}+k_{2}^{\mu} Y_{2}^{\mu}+k_{3}^{\mu} Y_{3}^{\mu}+\ldots\right)}
$$

\section{Level 1:}

Let us express $L$ in loop variable notation:

$$
L=\left(i k_{1}^{\mu} Y_{1}^{\mu}\right) e^{i k_{0} Y}
$$

The coefficient of $Y_{1}^{\mu}$ in the linear part of the ERG is

$$
\int d z \dot{G}(z, z)\left[\left(i k_{0}\right)^{2} i k_{1}^{\mu} Y_{1}^{\mu} e^{i k_{0} Y}-\frac{\partial}{\partial x_{1}}\left(i k_{0} . i k_{1} e^{i k_{0} Y}\right)\right]=\left[-\left(k_{0}\right)^{2} i k_{1}^{\mu} Y_{1}^{\mu}+\left(k_{0} . k_{1} i k_{0}^{\mu} Y_{1}^{\mu}\right)\right] e^{i k_{0} Y}
$$

This is clearly Maxwell's equation.

Note that in the ERG one can integrate by parts and let the derivatives act on $\dot{G}(z, z)$. In this case the LHS of (3.5.55) can be written as:

$$
\int d z\left[\dot{G}(z, z)\left[\left(i k_{0}\right)^{2} i k_{1}^{\mu} Y_{1}^{\mu} e^{i k_{0} Y}\right]+\frac{\partial \dot{G}(z, z)}{\partial x_{1}}\left[\left(i k_{0} . i k_{1} e^{i k_{0} Y}\right)\right]\right]
$$

If we let $G(z, z)=\Sigma$ the similarity with (3.2.42) is clear.

\section{Higher Levels:}

One can similarly look at the contribution of $k_{n} Y_{n} e^{i k_{0} Y}$ in $L$. It gives a contribution:

$$
\frac{\partial \dot{G}(z, z)}{\partial x_{n}}\left[\left(i k_{0} . i k_{n} e^{i k_{0} Y}\right)\right]
$$

to the linear term of the ERG. This is recognizable as $-\frac{\partial \Sigma}{\partial x_{n}} k_{0} \cdot k_{n} e^{i k_{0} Y}$.

Similarly consider $i k_{n} . Y_{n} i k_{m} . Y_{m} e^{i k_{0} Y}$ in the Lagrangian. The new contribution translated to the loop variable notation is after integration by parts:

$$
\int d z \int d z^{\prime} \dot{G}\left(z, z^{\prime}\right) \partial_{z} \partial_{z}^{\prime}\left[\frac{\partial^{2} L}{\partial X^{\prime}(z) \partial X^{\prime}\left(z^{\prime}\right)} \delta\left(z-z^{\prime}\right)\right]=-\int d z \int d z^{\prime} \frac{\partial^{2} \dot{G}\left(z, z^{\prime}\right)}{\partial x_{n} \partial x_{m}^{\prime}} k_{n} . k_{m} \delta\left(z-z^{\prime}\right)
$$

The delta function implies that

$$
\int d z \int d z^{\prime} \frac{\partial^{2} \dot{G}\left(z, z^{\prime}\right)}{\partial x_{n} \partial x_{m}^{\prime}} \delta\left(z-z^{\prime}\right)=\int d z\left\langle Y_{n}(z) Y_{m}(z)\right\rangle=\int d z \frac{1}{2}\left(\frac{\partial^{2}}{\partial x_{n} \partial x_{m}}-\frac{\partial}{\partial x_{n+m}}\right) \Sigma
$$

This should be compared with (3.2.42). Thus, as should have been expected, the linear part of the ERG reproduces the free equations of motion obtained in the usual loop variable approach reviewed in Section 3. The gauge invariance has already been argued in Section 3 and also explicitly demonstrated in earlier papers. We now turn to the interacting equations. 


\subsection{Quadratic Terms}

Letting $X(z), X\left(z^{\prime}\right)$ be $Y\left(z_{A}\right), Y\left(z_{B}\right)$ and $X^{\prime}(z)$ stand for $\frac{\partial Y}{\partial x_{n A}}$ and $X^{\prime}\left(z^{\prime}\right)$ stand for $\frac{\partial Y}{x_{m B}}$, for the various $x_{n}$ 's it is easy to see that each of the four quadratic terms stand for (after integrating by parts as in the linear case), respectively, terms of the form:

$$
\begin{gathered}
k_{0}(A) \cdot k_{0}(B) \dot{G}\left(z_{A}, z_{B}\right), \quad k_{n A} \cdot k_{0}(B) \frac{\partial \dot{G}\left(z_{A}, z_{B}\right)}{\partial x_{n A}}, \\
k_{n B} \cdot k_{0}(A) \frac{\partial \dot{G}\left(z_{A}, z_{B}\right)}{\partial x_{n B}}, \quad k_{n A} \cdot k_{m B} \frac{\partial^{2} \dot{G}\left(z_{A}, z_{B}\right)}{\partial x_{n A} \partial x_{m B}}
\end{gathered}
$$

The argument for gauge invariance works exactly as in the free case and involves showing that gauge transformations result in total derivatives. We need only worry about the fields labeled by $A$ as their transformation is completely independent of fields at $B$. Thus for instance: under $k_{n}(A) \rightarrow \lambda_{n A} k_{0}(A)$, the term $k_{n A} \cdot k_{0}(B) \frac{\partial \dot{G}\left(z_{A}, z_{B}\right)}{\partial x_{n A}} e^{i k_{0} Y}$ goes over to $\lambda_{n}(A) \frac{\partial}{\partial x_{n A}}\left[\dot{G}\left(z_{A}, z_{B}\right)\right] k_{0}(A) \cdot k_{0}(B) e^{i k_{0} Y}$. Similarly the term

$$
k_{n}(A) Y_{n}(A) e^{i k_{0} Y} k_{0}(A) \cdot k_{0}(B) \dot{G}\left(z_{A}, z_{B}\right)
$$

goes over to $\lambda_{n}(A) \frac{\partial}{\partial x_{n A}}\left[e^{i k_{0}(A) \cdot Y(A)}\right] k_{0}(A) \cdot k_{0}(B) \dot{G}\left(z_{A}, z_{B}\right)$. Thus the total change is of the form $\lambda_{n}(A) \frac{\partial}{\partial x_{n A}}\left[e^{i k_{0} Y} k_{0}(A) \cdot k_{0}(B) \dot{G}\left(z_{A}, z_{B}\right)\right]$, a total derivative. This guarantees that the equation obtained as the coefficient of $\dot{G}\left(z_{A}, z_{B}\right)$, which will involve integrating by parts on $x_{n A}$, will be gauge invariant under $k_{n}(A) \rightarrow \lambda_{n}(A) k_{0}(A)$. (The reader is encouraged to verify this!)

However there is a problem with the lower invariances of the form $k_{n}(A) \rightarrow$ $\lambda_{p A} k_{n-p}(A)$, for the term $k_{n A} \cdot k_{0}(B) \frac{\partial \dot{G}\left(z_{A}, z_{B}\right)}{\partial x_{n} A} e^{i k_{0} Y}$ becomes

$\lambda_{p}(A) \frac{\partial}{\partial x_{n A}}\left[\dot{G}\left(z_{A}, z_{B}\right)\right] k_{n-p}(A) \cdot k_{0}(B) e^{i k_{0} Y}$. Although this is actually equal to $\lambda_{n}(A) \frac{\partial^{2}}{\partial x_{p A} \partial x_{n-p A}}\left[\dot{G}\left(z_{A}, z_{B}\right)\right] k_{n-p}(A) \cdot k_{0}(B) e^{i k_{0} Y}$, the result of integration by parts is clearly not the same. The first form gives one derivative of $x_{n A}$ on the remaining terms, with a sign reversal, whereas the second version gives two derivatives of $x_{n-p, A}$ and $x_{p A}$, without a sign reversal. It is the second version that we need for invariance under $\lambda_{p}(A)$, whereas the first one gives invariance under $\lambda_{n}(A)$.

The resolution of this is to split $k_{n}$ into pieces, each of which transforms only under some of the $\lambda_{m}$. This will be described in the next section where explicit calculations are performed for level two and level three. 
To summarize this section, we have given the ERG in terms of loop variables. The linear part gives the gauge invariant free equation. The quadratic part gives the interacting part of the equation. However there is an issue regarding gauge invariance for which we have to find a solution. This is given explicitly for level two and three in the next section.

\section{Examples}

In this section we set the tachyon to zero, since there is no gauge invariance associated with it. We have already worked out the level 1 results, which gives the free Maxwell equation. We now turn to level 2.

\subsection{Level 2:}

\subsubsection{Linear Terms:}

The Lagrangian is:

$$
L=i k_{1}^{\mu} Y_{1}^{\mu} e^{i k_{0} Y}+i k_{2}^{\mu} Y_{2}^{\mu} e^{i k_{0} Y}-\frac{1}{2} k_{1}^{\mu} k_{1}^{\nu} Y_{1}^{\mu} Y_{1}^{\nu} e^{i k_{0} Y}
$$

The various terms are:

I.

$$
\iint d z d z^{\prime} \dot{G}\left(z, z^{\prime}\right)\left[\frac{\partial^{2} L}{\partial X(z)^{2}} \delta\left(z-z^{\prime}\right)\right]=\int d z \dot{G}(z, z)\left[i k_{0} . i k_{0} i k_{2}^{\mu} Y_{2}^{\mu}-\frac{1}{2} i k_{0} . i k_{0} k_{1}^{\mu} k_{1}^{\nu} Y_{1}^{\mu} Y_{1}^{\nu}\right] e^{i k_{0} Y}
$$

II.

$$
\begin{gathered}
\iint d z d z^{\prime} \dot{G}\left(z, z^{\prime}\right)\left[-\partial_{z}\left[\frac{\partial^{2} L}{\partial X(z) \partial X^{\prime}(z)}\right] \delta\left(z-z^{\prime}\right)\right]= \\
\int d z \dot{G}(z, z)\left[-\partial_{x_{2}}\left[i k_{2} . i k_{0} e^{i k_{0} Y}\right]+\partial_{x_{1}}\left[i k_{0} \cdot k_{1} k_{1}^{\nu} Y_{1}^{\nu} e^{i k_{0} Y}\right]\right] \\
=\int d z \dot{G}(z, z)\left[-i k_{2} . i k_{0} i k_{0}^{\mu} Y_{2}^{\mu}+i k_{0} \cdot k_{1}\left[k_{1}^{\mu} Y_{2}^{\mu}+k_{1}^{\nu} Y_{1}^{\nu} i k_{0} \cdot Y_{1}\right] e^{i k_{0} Y}\right.
\end{gathered}
$$

III.

$$
\iint d z d z^{\prime} \dot{G}\left(z, z^{\prime}\right) \partial_{z} \partial_{z^{\prime}}\left[\frac{\partial^{2} L}{\partial X^{\prime}(z)^{2}} \delta\left(z-z^{\prime}\right)\right]
$$


As an intermediate step we can write this as

$$
\begin{gathered}
\iint d z d z^{\prime} \partial_{z} \partial_{z^{\prime}}\left[\dot{G}\left(z, z^{\prime}\right)\right] \delta\left(z-z^{\prime}\right) \frac{\partial^{2} L}{\partial X^{\prime}(z)^{2}}=\int d z\left\langle Y_{1}(z) Y_{1}(z)\right\rangle \frac{\partial^{2} L}{\partial X^{\prime}(z)^{2}} \\
=\int d z \frac{1}{2}\left(\frac{\partial^{2}}{\partial_{x_{1}}^{2}}-\frac{\partial}{\partial_{x_{2}}}\right)[\dot{G}(z, z)] \frac{\partial^{2} L}{\partial X^{\prime}(z)^{2}}=\int d z \dot{G}(z, z) \frac{1}{2}\left(\frac{\partial^{2}}{\partial_{x_{1}}^{2}}+\frac{\partial}{\partial_{x_{2}}}\right) \frac{\partial^{2} L}{\partial X^{\prime}(z)^{2}} \\
=-\int d z \dot{G}(z, z) k_{1} \cdot k_{1}\left(i k_{0} \cdot Y_{2}+\frac{1}{2}\left(i k_{0} \cdot Y_{1}\right)^{2}\right) e^{i k_{0} Y}
\end{gathered}
$$

We can collect the coefficients of $Y_{2}^{\mu}$ :

$$
-\left(k_{0}\right)^{2} i k_{2}^{\mu}+k_{0} \cdot k_{2} i k_{0}^{\mu}+i k_{0} \cdot k_{1} k_{1}^{\mu}-k_{1} \cdot k_{1} i k_{0}^{\mu}
$$

The coefficients of $Y_{1}^{\mu} Y_{1}^{\nu}$ are:

$$
\frac{1}{2} k_{0}^{2} k_{1}^{\mu} k_{1}^{\nu}-\frac{1}{2} k_{0} \cdot k_{1} k_{1}^{(\mu} k_{0}^{\nu)}+\frac{1}{2} k_{0}^{\mu} k_{0}^{\nu} k_{1} \cdot k_{1}
$$

They are written as massless equations in one higher dimension. These are gauge invariant under $k_{2}^{\mu} \rightarrow k_{2}^{\mu}+\lambda_{2} k_{0}^{\mu}+\lambda_{1} k_{1}^{\mu}, \quad k_{1}^{\mu} \rightarrow k_{1}^{\mu}+\lambda_{1} k_{0}^{\mu}$.

After dimensional reduction they become

$$
\begin{gathered}
-k_{0}^{2} i k_{2}^{\mu}+k_{0} \cdot k_{2} i k_{0}^{\mu}+i k_{0} \cdot k_{1} k_{1}^{\mu}-k_{1} \cdot k_{1} i k_{0}^{\mu}=0 \\
\frac{1}{2}\left(k_{0}^{2}+q_{0}^{2}\right) k_{1}^{\mu} k_{1}^{\nu}-\frac{1}{2} k_{0} \cdot k_{1} k_{1}^{(\mu} k_{0}^{\nu)}-\frac{1}{2} q_{0}^{2} k_{2}^{(\mu} k_{0}^{\nu)}+\frac{1}{2} k_{0}^{\mu} k_{0}^{\nu} k_{1} \cdot k_{1}+\frac{1}{2} k_{0}^{\mu} k_{0}^{\nu} q_{2} q_{0}=0 \\
-k_{0}^{2} i q_{2}+2 k_{0} \cdot k_{2} i q_{0}-k_{1} \cdot k_{1} i q_{0}=0
\end{gathered}
$$

The gauge transformation law for $q_{2}$ is $\delta q_{2}=2 \lambda_{2} q_{0}$.

As explained earlier the canonical dimension of the operator is obtained as $q_{0}^{2}$ in this formalism. Thus where, in the OC formalism, in the LHS of the ERG (2.2.16), i.e. $\frac{\partial L}{\partial \tau}$, we had both the contribution of the canonical scaling, and the $\beta$-function (see (2.3.19) ), now we need only the beta function. The canonical dimension that gives the tree level mass shows up in the RHS of the ERG in the form of the "anomalous" term $q_{0}^{2} 6$

\footnotetext{
${ }^{6}$ This is why in the loop variable formalism, string theory looks like a massless theory in one higher dimension.
} 


\subsubsection{Quadratic Terms}

The contribution of quadratic terms to level 2 can come from various sources. It can come from level 1 as well as level 2 . We need to calculate $\frac{\partial L}{\partial X(z)}-$ $\partial_{z} \frac{\partial L}{\partial X^{\prime}(z)}$ and thence

$$
\begin{gathered}
\iint d z_{A} d z_{B} \dot{G}\left(z_{A}, z_{B}\right)\left[\frac{\partial L\left[X\left(z_{A}\right), X^{\prime}\left(z_{A}\right)\right]}{\partial X\left(z_{A}\right)}-\partial_{z_{A}} \frac{\partial L\left[X\left(z_{A}\right), X^{\prime}\left(z_{A}\right)\right]}{\partial X^{\prime}\left(z_{A}\right)}\right] \\
{\left[\frac{\partial L\left[X\left(z_{B}\right), X^{\prime}\left(z_{B}\right)\right]}{\partial X\left(z_{B}\right)}-\partial_{z_{B}} \frac{\partial L\left[X\left(z_{B}\right), X^{\prime}\left(z_{B}\right)\right]}{\partial X^{\prime}\left(z_{B}\right)}\right]}
\end{gathered}
$$

The mechanism of gauge invariance discussed in the previous section suggests that under the gauge transformation $k_{2}^{\mu}(A) \rightarrow k_{2}^{\mu}(A)+\lambda_{2}(A) k_{0}^{\mu}(A)+$ $\lambda_{1}(A) k_{1}^{\mu}(A), \quad k_{1}^{\mu}(A) \rightarrow \lambda_{1}(A) k_{0}^{\mu}(A)$, if this expression is to be invariant then each of the two factors in the product should be invariant. But one can check that this is not so. Consider the level 2 term

$$
\partial_{x_{1}} \frac{\partial L\left[Y\left(z_{A}\right), Y_{1}\left(z_{A}\right)\right]}{\partial Y_{1}\left(z_{A}\right)}=-\left(k_{1}^{\mu} k_{1}^{\nu} Y_{2}^{\nu}+k_{1}^{\mu} k_{1} . Y_{1} i k_{0} \cdot Y_{1}\right) e^{i k_{0} Y}
$$

One of the terms in the gauge variation is $\lambda_{1} k_{1}^{\mu} k_{0} \cdot Y_{1} i k_{0} \cdot Y_{1} e^{i k_{0} Y}$. It can easily be checked that there is no term that can cancel this. What ensures gauge invariance is that the gauge variation of every term in the Lagrangian should be a derivative of some lower level term. Thus the $\lambda_{1}$ variation of level two should give $\lambda_{1} \partial_{x_{1}} k_{1} \cdot Y_{1}=\lambda_{1} k_{1} \cdot \partial_{x_{1}}^{2} Y$. Although $\partial_{x_{1}}^{2} Y=Y_{2}$ there is a difference between the two. This distinction is important because $\frac{\partial^{2}}{\partial x_{1}^{2}}$ is not identically equal to $\frac{\partial}{\partial x_{2}}$ - it is only so when acting on $Y$. In particular one gets different results when integrating by parts. The end result now is gauge invariant. Thus we need to find a combination of loop variables that gives only $\lambda_{1}$ and this should be the coefficient of $\partial_{x_{1}}^{2} Y$. This combination is

$$
K_{11}^{\mu} \equiv k_{2}^{\mu}-Q_{2} k_{0}^{\mu} \equiv k_{2}^{\mu}-\left(q_{2}-\frac{q_{1}^{2}}{2 q_{0}}\right) k_{0}^{\mu}
$$

with gauge transformation:

$$
\delta K_{11}^{\mu}=\lambda_{1} k_{1}^{\mu} \quad ; \quad \delta Q_{2} k_{0}^{\mu}=\lambda_{2} k_{0}^{\mu}
$$

If we use $q_{1} q_{1}=q_{2} q_{0}$ then $Q_{2}=\frac{1}{2} q_{2}$. Thus the strategy is to write $K_{11}^{\mu} \partial_{x_{1}}^{2} Y^{\mu}+Q_{2} k_{0}^{\mu} Y_{2}^{\mu}$ instead of $k_{2}^{\mu} Y_{2}^{\mu}$.

We work out the consequences of this explicitly now. 
First modify the form of the ERG to accommodate second derivatives in $L\left[X, X^{\prime}, X^{\prime \prime}\right]$ and we get $\frac{\partial L}{\partial X(z)}-\partial_{z} \frac{\partial L}{\partial X^{\prime}(z)}+\partial_{z}^{2} \frac{\partial L}{\partial X^{\prime \prime}(z)}$. Let us evaluate this for the Lagrangian 7 :

$$
\begin{gathered}
L=\left[i\left(k_{2}^{\mu}-\frac{1}{2} q_{2} k_{0}^{\mu}\right) \frac{\partial^{2} Y^{\mu}}{\partial x_{1}^{2}}+\frac{1}{2} i q_{2} k_{0}^{\mu} \frac{\partial Y^{\mu}}{\partial x_{2}}-\frac{1}{2} k_{1}^{\mu} k_{1}^{\nu} Y_{1}^{\mu} Y_{1}^{\nu}\right] e^{i k_{0} Y} \\
\frac{\partial L}{\partial Y^{\mu}}=\left[i k_{0}^{\mu} i\left(k_{2}^{\mu}-\frac{1}{2} q_{2} k_{0}^{\mu}\right) \frac{\partial^{2} Y^{\mu}}{\partial x_{1}^{2}}+i k_{0}^{\mu} \frac{1}{2} i q_{2} k_{0}^{\mu} \frac{\partial Y^{\mu}}{\partial x_{2}}-i k_{0}^{\mu} \frac{1}{2} k_{1}^{\mu} k_{1}^{\nu} Y_{1}^{\mu} Y_{1}^{\nu}\right] e^{i k_{0} Y} \\
\partial_{x_{1}} \frac{\partial L}{\partial Y_{1}^{\mu}}=-k_{1}^{\mu} k_{1} \cdot Y_{2} e^{i k_{0} Y}-k_{1}^{\mu} k_{1} \cdot Y_{1} i k_{0} \cdot Y_{1} e^{i k_{0} Y} \\
\partial_{x_{2}} \frac{\partial L}{\partial Y_{2}^{\mu}}=\frac{1}{2} i q_{2} k_{0}^{\mu} i k_{0} \cdot Y_{2} e^{i k_{0} Y} \\
\partial_{x_{1}}^{2} \frac{\partial L}{\partial\left(\partial_{x_{1}}^{2} Y^{\mu}\right)}=i\left(k_{2}^{\mu}-\frac{1}{2} q_{2} k_{0}^{\mu}\right)\left(i k_{0} \cdot Y_{2}+\left(i k_{0} \cdot Y_{1}\right)^{2}\right) e^{i k_{0} Y}
\end{gathered}
$$

Thus

$$
\begin{gathered}
\frac{\partial L}{\partial X(z)}-\partial_{z} \frac{\partial L}{\partial X^{\prime}(z)}+\partial_{z}^{2} \frac{\partial L}{\partial X^{\prime \prime}(z)}=\left[i k_{0}^{\mu} i\left(k_{2}^{\mu}-\frac{1}{2} q_{2} k_{0}^{\mu}\right) \frac{\partial^{2} Y^{\mu}}{\partial x_{1}^{2}}+i k_{0}^{\mu} \frac{1}{2} i q_{2} k_{0}^{\mu} \frac{\partial Y^{\mu}}{\partial x_{2}}-i k_{0}^{\mu} \frac{1}{2} k_{1}^{\mu} k_{1}^{\nu} Y_{1}^{\mu} Y_{1}^{\nu}\right] e^{i k_{0} Y} \\
+\left(k_{1}^{\mu} k_{1} \cdot Y_{2} e^{i k_{0} Y}+k_{1}^{\mu} k_{1} \cdot Y_{1} i k_{0} \cdot Y_{1} e^{i k_{0} Y}\right)-\frac{1}{2} i q_{2} k_{0}^{\mu} i k_{0} \cdot Y_{2} e^{i k_{0} Y} \\
+i\left(k_{2}^{\mu}-\frac{1}{2} q_{2} k_{0}^{\mu}\right)\left(i k_{0} \cdot Y_{2}+\left(i k_{0} \cdot Y_{1}\right)^{2}\right) e^{i k_{0} Y}
\end{gathered}
$$

In (4.1.66) one can replace $\frac{\partial^{2} Y^{\mu}}{\partial x_{1}^{2}}$ by $\frac{\partial Y^{\mu}}{\partial x_{2}}$. This expression is gauge invariant as can easily be checked explicitly. The coefficient of $Y_{2}^{\nu}$ is :

$$
V_{2}^{\mu \nu} \equiv\left[-k_{0}^{\mu} k_{2}^{\nu}+k_{1}^{\mu} k_{1}^{\nu}-k_{2}^{\mu} k_{0}^{\nu}+q_{2} k_{0}^{\mu} k_{0}^{\nu}\right]
$$

The coefficient of $Y_{1}^{\mu} Y_{1}^{\nu}$ is:

$$
V_{11}^{\rho \mu \nu} \equiv\left[-\frac{1}{2} k_{0}^{\rho} k_{1}^{\mu} k_{1}^{\nu}+\frac{1}{2} k_{1}^{\rho}\left(k_{1}^{\mu} k_{0}^{\nu}+k_{1}^{\nu} k_{0}^{\mu}\right)-\left(k_{2}^{\rho}-\frac{1}{2} q_{2} k_{0}^{\rho}\right) k_{0}^{\mu} k_{0}^{\nu}\right]
$$

\footnotetext{
${ }^{7}$ Note that this Lagrangian is identical to the earlier one used for the linear part. The rewriting only has the effect of generating a different set of terms when one integrates by parts. Thus the total derivatives that are being added or dropped are different. This is thus a physically equivalent Lagrangian.
} 
The $V$ 's defined above are gauge invariant and are the analogues of the field strength $F^{\mu \nu} \equiv k_{0}^{\mu} k_{1}^{\nu}-k_{0}^{\nu} k_{1}^{\mu}$ for the photon. If we define

$$
L_{1}^{\mu}(z) \equiv F^{\mu \rho} Y_{1}^{\rho}(z) e^{i k_{0} . Y(z)}
$$

and

$$
L_{2}^{\mu}(z) \equiv\left[V_{2}^{\mu \rho} Y_{2}^{\rho}(z)+V_{11}^{\mu \rho \sigma} Y_{1}^{\rho}(z) Y_{1}^{\sigma}(z)\right] e^{i k_{0} . Y(z)}
$$

Thus the quadratic terms in the ERG takes the following form

$$
\begin{gathered}
\int d z_{A} \int d z_{B} \dot{G}\left(z_{A}, z_{B}\right)\left[\left(L_{1}^{\mu}\left(z_{A}\right)+L_{2}^{\mu}\left(z_{A}\right)\right)\left(L_{1}^{\mu}\left(z_{B}\right)+L_{2}^{\mu}\left(z_{B}\right)\right)\right]= \\
\int d z_{A} \int d z_{B} \dot{G}\left(z_{A}, z_{B}\right)\left[F_{\rho \nu} Y_{1}^{\nu}\left(z_{A}\right)+V_{2 \rho \nu} Y_{2}^{\nu}\left(z_{A}\right)+V_{11 \rho \mu \nu} Y_{1}^{\mu}\left(z_{A}\right) Y_{1}^{\nu}\left(z_{A}\right)\right] e^{i k_{0}(A) . Y\left(z_{A}\right)} \\
{\left[F_{\alpha}^{\rho} Y_{1}^{\alpha}\left(z_{B}\right)+V_{2}^{\rho} Y_{2}^{\alpha}\left(z_{B}\right)+V_{11 \alpha \beta}^{\rho} Y_{1}^{\alpha}\left(z_{B}\right) Y_{1}^{\beta}\left(z_{B}\right)\right] e^{i k_{0}(B) . Y\left(z_{B}\right)}}
\end{gathered}
$$

Before combining with the linear term (4.1.63, 4.1.62) one needs to perform the OPE's in (4.1.69).

\subsubsection{Operator Product Expansion}

Using the results of Appendix B we can perform the OPE's. The linear term in the ERG evaluates the contribution from self contractions within a vertex operator. So we can assume that the vertex operators are normal ordered for the purposes of the calculation of the quadratic term. The contractions indicated by the general formula in Appendix B for quadratic terms involves contraction between fields at different points and is different from the self contractions of normal ordering. Thus in performing the OPE one can assume that they are normal ordered.

Now we can use the results of Appendix B to write down the OPE between these terms. Thus for instance to use the formulae there, $k_{1}^{\nu}, p_{1}^{\nu}$ there will stand for $F^{\mu \nu}$ and $k_{1}^{\rho} k_{1}^{\nu}, p_{1}^{\rho} p_{1}^{\nu}$ will stand for $V_{11}^{\mu \rho \nu}$. After all the substitutions are made one gets interacting equations of motion for the combined level 1, level 2 system (and level 0 if we include the tachyon).

The full result involves a large number of terms and is not very illuminating. We give a sampling of some of the terms below:

Contribution of level 2 (massive spin 2) and level 1 (photon) to Maxwell's equation

$$
\int d z_{A} \dot{G}\left(z_{A}, z_{A}\right) i \partial_{\nu} F^{\mu \nu}+\int d z_{A} \int d z_{B} \dot{G}\left(z_{A}, z_{B}\right)\left[i \frac{1}{2}\left(\partial_{\rho} \partial_{\sigma} F^{\nu \mu}\right) V_{11}^{\nu \rho \sigma}\left(G_{1,0}\right)^{2}+\right.
$$




$$
\begin{gathered}
i \frac{1}{2}\left(z_{B}-z_{A}\right)\left(\partial_{\lambda} V_{11 \nu}^{\rho \sigma}\right)\left(\partial^{\mu} \partial_{\rho} \partial_{\sigma} F^{\nu \lambda}\right)\left(G_{1,0}\right)^{2} \\
-i G_{1,0}^{2} G_{0,1}\left(\partial_{\lambda} V_{11}^{\alpha \rho \sigma}\right)\left(\partial_{\rho} \partial_{\sigma} V_{11 \alpha}^{\lambda \mu}\right)+\frac{i}{4}\left(z_{B}-z_{A}\right) G_{0,1}^{2} G_{1,0}^{2}\left(\partial_{\alpha} \partial_{\beta} V_{11}^{\lambda \rho \sigma}\right)\left(\partial_{\rho} \partial_{\sigma} \partial^{\mu} V_{11 \lambda}^{\alpha \beta}\right)+ \\
-i G_{1,1} F^{\nu \sigma} V_{11}^{\nu \sigma \mu}+i\left(z_{2}-z_{1}\right) G_{1,1} G_{1,0} \partial^{\mu} \partial^{\rho} F^{\nu \sigma} V_{11}^{\nu \rho \sigma}+ \\
i \frac{\left(z_{2}-z_{1}\right)}{2} G_{1,1}^{2}\left(\partial^{\mu} V_{11}^{\nu \rho \sigma}\right) V_{11 \nu \rho \sigma}+2 i G_{1,1} G_{1,0} V_{11}^{\nu \rho \sigma} \partial_{\sigma} V_{11 \nu \rho}{ }^{\mu} \\
\left.-i\left(z_{2}-z_{1}\right) G_{1,1} G_{1,0} G_{0,1}\left(\partial^{\mu} \partial^{\lambda} V_{11}^{\nu \rho \sigma}\right) \partial_{\sigma} V_{11 \nu \rho \lambda}+\ldots\right]=0
\end{gathered}
$$

The argument of the Green function, $z_{A}-z_{B}$, has been suppressed. The three dots represent contribution from other fields.

Some contributions of level 2 (massive spin 2) and level 1 to level 2 equation

$$
\begin{aligned}
& \int d z_{A} \dot{G}\left(z_{A}, z_{A}\right)\left[\frac{1}{2}\left(\partial^{2}-1\right) S_{11}^{\mu \nu}-\frac{1}{2} \partial_{\rho} \partial^{(\nu} S_{11}^{\mu) \rho}+\frac{1}{2} \partial^{(\mu} S_{2}^{\mu)}+\frac{1}{2} \partial^{\mu} \partial^{\nu} S_{11 \rho}^{\rho}-\frac{1}{2} \partial^{\mu} \partial^{\nu} S_{2}\right]+ \\
& \int d z_{A} \int d z_{B} \dot{G}\left(z_{A}, z_{B}\right)\left[V_{11}^{\lambda \rho \mu}\left(\partial_{\rho} F^{\lambda \nu}\right) G_{10}-\left(z_{B}-z_{A}\right) V_{11}^{\lambda \rho \sigma}\left(\partial_{\rho} \partial_{\sigma} \partial^{\nu} F_{\lambda}^{\mu}\right) \frac{G_{10}^{2}}{2}+\partial_{\rho} V_{11}^{\lambda \mu \nu} F_{\lambda}^{\rho} \frac{G_{0,1}}{2}\right. \\
& -\left(z_{B}-z_{A}\right) \partial_{\sigma} V_{11}^{\lambda \rho \mu} \partial^{\nu} \partial_{\rho} F_{\lambda}^{\sigma}\left(G_{1,0} G_{0,1}\right)+\frac{\left(z_{B}-z_{A}\right)^{2}}{4}\left(\partial_{\delta} V_{11}^{\lambda \rho \sigma}\right)\left(\partial_{\rho} \partial_{\sigma} \partial^{\mu} \partial^{\nu} F_{\lambda}^{\delta}\right) G_{1,0}^{2} G_{0,1} \\
& +G_{1,1} V_{11}^{\sigma \rho \mu} V_{11 \sigma \rho}{ }^{\nu}-\frac{\left(z_{2}-z_{1}\right)^{2}}{2} G_{1,1}^{2}\left(\partial^{\mu} \partial^{\nu} V_{11}^{\lambda \rho \sigma}\right) V_{11 \lambda \rho \sigma} \\
& -G_{1,0}^{2}\left(V_{11}^{\lambda \rho \sigma}\right)\left(\partial_{\rho} \partial_{\sigma} V_{11 \lambda}^{\mu \nu}\right)-G_{1,0}^{2}\left(\partial_{\sigma} V_{11}^{\lambda \rho \mu}\right)\left(\partial_{\rho} V_{\lambda}^{\sigma \nu}\right)+\frac{\left(z_{B}-z_{A}\right)}{2} G_{1,0}^{2} G_{0,1}\left(\partial_{\alpha} V_{11}^{\lambda \rho \sigma}\right)\left(\partial^{\nu} \partial_{\rho} \partial_{\sigma} V_{\lambda}^{\alpha \mu}\right) \\
& +\frac{\left(z_{B}-z_{A}\right)}{2} G_{1,0} G_{0,1}^{2}\left(\partial^{\nu} \partial_{\alpha} V_{11}^{\lambda \rho \sigma}\right)\left(\partial_{\rho} \partial_{\sigma} V_{\lambda}^{\alpha \mu}\right)-\frac{\left(z_{B}-z_{A}\right)^{2}}{2}\left(\partial_{\alpha} \partial_{\beta} V_{11}^{\lambda \rho \sigma}\right)\left(\partial^{\mu} \partial^{\nu} \partial_{\rho} \partial_{\sigma} V_{11 \lambda}^{\alpha \beta}\right) \frac{G_{1,0}^{2} G_{0,1}^{2}}{4}+ \\
& +\left(z_{2}-z_{1}\right) G_{1,1} G_{1,0}\left(\partial^{\nu} \partial^{\rho} V_{11}^{\lambda \sigma \mu}\right) V_{11 \lambda \sigma \rho}-\left(z_{2}-z_{1}\right) G_{1,1} G_{1,0}\left(\partial^{\nu} V^{\lambda \rho \sigma}\right)\left(\partial_{\sigma} V_{11 \lambda \rho}{ }^{\mu}\right) \\
& \left.-\frac{\left(z_{2}-z_{1}\right)^{2}}{2} G_{1,1} G_{1,0} G_{0,1}\left(\partial^{\tau} V^{\lambda \sigma \rho}\right)\left(\partial^{\mu} \partial^{\nu} \partial_{\sigma} V_{11 \lambda \rho \tau}\right)+\ldots\right]=0
\end{aligned}
$$

where

$$
\begin{gathered}
V_{11}^{\mu \rho \sigma}=i\left[-\frac{\partial^{\mu} S_{11}^{\rho \sigma}}{2}+\frac{\partial^{(\rho} S_{11}^{\sigma) \mu}}{2}-\partial^{\rho} \partial^{\sigma} S_{2}^{\mu}+\frac{1}{2} \partial^{\mu} \partial^{\rho} \partial^{\sigma} S_{2}\right] \\
V_{2}^{\mu \nu}=-\partial^{(\mu} S_{2}^{\nu)}+S_{11}^{\mu \nu}-\partial^{\mu} \partial^{\nu} S_{2}
\end{gathered}
$$

are the gauge invariant field strengths. In the above only the contribution from $V_{11}$ is given.

We reproduce the gauge transformations of the fields

$$
\delta S_{11}^{\mu \nu}=\partial^{(\mu} \Lambda_{11}^{\nu)} \quad ; \quad \delta S_{2}^{\mu}=\Lambda_{11}^{\mu}+\partial^{\mu} \Lambda_{2} \quad ; \quad \delta S_{2}=2 q_{0} \Lambda_{2}
$$

Note that $q_{0}$ has been set to 1 . 


\subsubsection{Dimensional Reduction}

We need to comment on the role of the $\mathrm{D}+1$ the coordinate and dimensional reduction. As explained in Sec 4.1.1, the role of $q_{0}$ is the give a mass to the fields in accordance with string theory spectrum. This requires that $q_{0}^{2}$ be set equal to the canonical dimension of the operator. The value of $q_{0}$ is thus fixed when the free equations are written down. Note that $V_{11}^{\mu \nu 5}=-V_{11}^{5 \mu \nu}=$ $\frac{i q_{0}}{2} V_{2}^{\mu \nu}$ and $V_{2}^{\mu 5}=0$, where ' 5 ' is symbolic for the 27 th extra dimension called $\theta$ in this paper.

Thus we need $G^{\theta \theta}(z, z)=G^{X X}(z, z)$, in order that the anomalous dimension come out as $k_{0 \mu} k_{0}^{\mu}=k_{0}^{2}+q_{0}^{2}$ in the linear part of the ERG. However we do not want contributions from $q_{0}$ in correlation functions between vertex operators at different locations. This would affect the pole structure of the S-matrix. Thus we want $G^{\theta \theta}\left(z, z^{\prime}\right) \rightarrow 0$ when $z \neq z^{\prime}$. This can be achieved by making the $\theta$ coordinate massive - with a mass of the order of the UV cutoff. Thus we take

$$
\left\langle\theta(z) \theta\left(z^{\prime}\right)\right\rangle=\int d^{2} q e^{i q \cdot\left(z-z^{\prime}\right)} \frac{1}{q^{2}+m^{2}}
$$

with $m \approx \frac{1}{a}$ where $a$ is the short distance cutoff or the lattice spacing.

Note that this implies that there is no translation invariance in the $\theta$ direction and there is no $q_{0}$-momentum conservation. The value of $q_{0}$ when it occurs in a field is fixed once and for all by the linearized theory. In computations, this means that in the ERG, the linear term gets a contribution from $\theta$ contractions in the normal ordering, but in the quadratic term the sum over $\mu$ does not include $\theta$. $\theta$ will continue to appear in the vertex operators for external states.

The propagator (4.1.70) would violate conformal invariance on the world sheet. However it does not affect the S-matrix for physical states or the space-time gauge invariance of the theory. The S- matrix is not affected because it has been argued [22] that the world sheet interaction Lagrangian for physical external states in the loop variable formalism reduces to that of the Lagrangian of the "Old Covariant" formalism with physical state constraints 8 Therefore if $\theta$ does not affect the correlation functions, the equivalence of the S-matrix follows. Space-time gauge invariance is not affected because this is built into the loop variable formalism and does not rely on world sheet symmetries.

\footnotetext{
${ }^{8}$ We caution that the demonstration has been explicitly done only for the second and third massive levels.
} 
The interactions are manifestly invariant under the same gauge transformations that leave the linear term invariant, i.e. the gauge transformation is not modified by the interactions. This is different from BRST string field theory where the gauge transformations are modified by the interactions and only the full equations of motion are invariant. In this sense we have an Abelian theory rather than a non-Abelian theory. It is possible that some field redefinitions in the BRST string field theory formulation will make it equivalent to this one. We also note that if we introduce Chan-Paton factors, the gauge transformations as well as the interactions will be modified in this formalism also.

\subsection{Level 3}

\subsubsection{Linear Terms}

The Lagrangian is:

$$
L=\left[i k_{3}^{\mu} Y_{3}^{\mu}-k_{2}^{\mu} k_{1}^{\nu} Y_{2}^{\mu} Y_{1}^{\nu}-\frac{i}{3 !} k_{1}^{\mu} k_{1}^{\nu} k_{1}^{\rho} Y_{1}^{\mu} Y_{1}^{\nu} Y_{1}^{\rho}\right] e^{i k_{0} Y}
$$

I.

The first term in the ERG is $\int d z \dot{G}(z, z) \frac{\partial^{2} L}{\partial X(z)^{2}}$ which gives

$$
\int d z \dot{G}(z, z)\left(-k_{0}^{2}\right)\left[i k_{3}^{\mu} Y_{3}^{\mu}-k_{2}^{\mu} k_{1}^{\nu} Y_{2}^{\mu} Y_{1}^{\nu}-\frac{i}{3 !} k_{1}^{\mu} k_{1}^{\nu} k_{1}^{\rho} Y_{1}^{\mu} Y_{1}^{\nu} Y_{1}^{\rho}\right] e^{i k_{0} Y}
$$

II.

The next term is: $-\int d z \dot{G}(z, z) \partial_{z} \frac{\partial^{2} L}{\partial X(z) \partial X^{\prime}(z)}$. Using

$\frac{\partial^{2} L}{\partial Y_{0}^{\mu}(z) \partial Y_{1}^{\mu}(z)}=i k_{0}^{\mu}\left[-\left(k_{2} \cdot Y_{2}\right) k_{1}^{\mu}-\frac{i}{2 !} k_{1}^{\mu}\left(k_{1} \cdot Y_{1}\right)^{2}\right] e^{i k_{0} Y}=\left[-\left(k_{2} \cdot Y_{2}\right) i k_{0} \cdot k_{1}+\frac{1}{2} k_{0} \cdot k_{1}\left(k_{1} \cdot Y_{1}\right)^{2}\right] e^{i k_{0} Y}$

we get:

$$
\begin{gathered}
\partial_{x_{1}} \frac{\partial^{2} L}{\partial Y_{0}^{\mu} \partial Y_{1}^{\mu}(z)}= \\
{\left[-k_{2} \cdot Y_{3} i k_{0} \cdot k_{1}+k_{0} \cdot k_{1} k_{1} \cdot Y_{1} k_{1} \cdot Y_{2}\right] e^{i k_{0} Y}+i k_{0} \cdot Y_{1}\left[-\left(k_{2} \cdot Y_{2}\right) i k_{0} \cdot k_{1}+\frac{1}{2} k_{0} \cdot k_{1}\left(k_{1} \cdot Y_{1}\right)^{2}\right] e^{i k_{0} Y}}
\end{gathered}
$$

Similarly

$$
\begin{gathered}
\frac{\partial^{2} L}{\partial Y_{0}^{\mu}(z) \partial Y_{2}^{\mu}(z)}=i k_{0}^{\mu}\left[-\left(k_{1} \cdot Y_{1}\right) k_{2}^{\mu}\right] e^{i k_{0} Y}=-i\left(k_{0} \cdot k_{2}\right)\left(k_{1} \cdot Y_{1}\right) k_{0} \cdot Y_{2} e^{i k_{0} Y} \\
\partial_{x_{2}} \frac{\partial^{2} L}{\partial Y_{0}^{\mu}(z) \partial Y_{2}^{\mu}(z)}=\left[-i\left(k_{0} \cdot k_{2}\right) k_{1} \cdot Y_{3}+\left(k_{0} \cdot k_{1}\right)\left(k_{1} \cdot Y_{1}\right)\left(k_{0} \cdot Y_{2}\right)\right] e^{i k_{0} Y}
\end{gathered}
$$


and

$$
\partial_{x_{3}} \frac{\partial^{2} L}{\partial Y_{0}^{\mu}(z) \partial Y_{3}^{\mu}(z)}=-i k_{0} \cdot k_{3}\left(k_{0} \cdot Y_{3}\right) e^{i k_{0} Y}
$$

III.

The last term is $\iint d z d z^{\prime} \partial_{z} \partial_{z^{\prime}} \dot{G}\left(z, z^{\prime}\right) \delta\left(z-z^{\prime}\right) \frac{\partial^{2} L}{\partial_{z} X^{\prime}(z)^{2}}$ which gives

$$
\begin{aligned}
& \frac{1}{2}\left(\frac{\partial^{2}}{\partial_{x_{1}}^{2}}-\frac{\partial}{\partial_{x_{2}}}\right) \dot{G}(z, z) \frac{\partial^{2} L}{\partial Y_{1}^{\mu} \partial Y_{1}^{\mu}}+\left(\frac{\partial^{2}}{\partial_{x_{1}} \partial_{x_{2}}}-\frac{\partial}{\partial_{x_{3}}}\right) \dot{G}(z, z) \frac{\partial^{2} L}{\partial Y_{1}^{\mu} \partial Y_{2}^{\mu}} \\
& \frac{\partial^{2} L}{\partial Y_{1}^{\mu} \partial Y_{1}^{\mu}}=-i k_{1} \cdot k_{1}\left(k_{1} \cdot Y_{1}\right) e^{i k_{0} Y} \quad ; \quad \frac{\partial^{2} L}{\partial Y_{1}^{\mu} \partial Y_{2}^{\mu}}=-k_{2} \cdot k_{1} e^{i k_{0} Y}
\end{aligned}
$$

Integrating by parts we get

$$
\begin{gathered}
\frac{1}{2}\left(\frac{\partial^{2}}{\partial_{x_{1}}^{2}}+\frac{\partial}{\partial_{x_{2}}}\right)\left[-i k_{1} \cdot k_{1}\left(k_{1} \cdot Y_{1}\right) e^{i k_{0} Y}\right]= \\
-\frac{i}{2}\left[k_{1} \cdot k_{1} 2\left(k_{1} \cdot Y_{3}+k_{1} \cdot Y_{2} i k_{0} \cdot Y_{1}+k_{1} \cdot Y_{1} k_{0} \cdot Y_{2}\right)-\frac{i}{2} k_{1} \cdot k_{1} k_{1} \cdot Y_{1}\left(i k_{0} \cdot Y_{1}\right)^{2}\right] e^{i k_{0} Y} \\
\left(\frac{\partial^{2}}{\partial_{x_{1}} \partial_{x_{2}}}+\frac{\partial}{\partial_{x_{3}}}\right)\left[-k_{2} \cdot k_{1} e^{i k_{0} Y}\right]=-k_{2} \cdot k_{1}\left[2 i k_{0} \cdot Y_{3}+i k_{0} \cdot Y_{1} i k_{0} \cdot Y_{2}\right] e^{i k_{0} Y}
\end{gathered}
$$

Adding the contributions of I, II, III we get

$$
\begin{aligned}
& \mathbf{Y}_{\mathbf{3}}^{\mu}\left[-k_{0}^{2} i k_{3}^{\mu}+i k_{0} \cdot k_{1} k_{2}^{\mu}+i k_{0} \cdot k_{2} k_{1}^{\mu}+i k_{0} \cdot k_{3} k_{0}^{\mu}-i k_{1} \cdot k_{1} k_{1}^{\mu}-2 i k_{1} \cdot k_{2} k_{0}^{\mu}\right] \\
+ & \mathbf{Y}_{\mathbf{2}}^{\mu} \mathbf{Y}_{\mathbf{1}}^{\nu}\left[k_{0}^{2} k_{2}^{\mu} k_{1}^{\nu}-k_{0} \cdot k_{1} k_{1}^{\mu} k_{1}^{\nu}-k_{0} \cdot k_{1} k_{2}^{\mu} k_{0}^{\nu}-k_{0} \cdot k_{2} k_{0}^{\mu} k_{1}^{\nu}+k_{1} \cdot k_{1} k_{0}^{\mu} k_{1}^{\nu}+k_{1} \cdot k_{1} k_{1}^{\mu} k_{0}^{\nu}+k_{1} \cdot k_{2} k_{0}^{\mu} k_{0}^{\nu}\right] \\
& +\mathbf{Y}_{\mathbf{1}}^{\mu} \mathbf{Y}_{\mathbf{1}}^{\nu} \mathbf{Y}_{\mathbf{1}}^{\rho}\left[\frac{i}{3 !} k_{0}^{2} k_{1}^{\mu} k_{1}^{\nu} k_{1}^{\rho}-\frac{i}{2} k_{0} \cdot k_{1} k_{1}^{\mu} k_{1}^{\nu} k_{0}^{\rho}+\frac{i}{2} k_{1} \cdot k_{1} k_{1}^{\mu} k_{0}^{\nu} k_{0}^{\rho}\right]=0
\end{aligned}
$$

These are the linear Spin 3 equations. They are gauge invariant under

$$
k_{3}^{\mu} \rightarrow k_{3}^{\mu}+\lambda_{3} k_{0}^{\mu}+\lambda_{2} k_{1}^{\mu}+\lambda_{1} k_{2}^{\mu}, \quad k_{2}^{\mu} \rightarrow k_{2}^{\mu}+\lambda_{2} k_{0}^{\mu}+\lambda_{1} k_{1}^{\mu}, \quad k_{1}^{\mu} \rightarrow k_{1}^{\mu}+\lambda_{1} k_{0}^{\mu}
$$

Note that they are written as massless higher dimensional equations. Mass can be introduced by dimensional reduction in the usual way. When this is done and the substitutions given in (3.3.50) are made we get 9:

$$
\begin{gathered}
\mathbf{Y}_{\mathbf{3}}^{\mu}\left[-k_{0}^{2} k_{3}^{\mu}+k_{0} \cdot k_{1} k_{2}^{\mu}+k_{0} \cdot k_{2} k_{1}^{\mu}+k_{0} \cdot k_{3} k_{0}^{\mu}-k_{1} \cdot k_{1} k_{1}^{\mu}-2 k_{1} \cdot k_{2} k_{0}^{\mu}-5 q_{3} q_{0} k_{0}^{\mu}\right]+ \\
\mathbf{Y}_{\mathbf{2}}^{\mu} \mathbf{Y}_{\mathbf{1}}^{\nu}\left[\frac{1}{2} k_{0}^{2} k_{2}^{(\mu} k_{1}^{\nu)}+\frac{1}{2}\left(k_{0}^{2}+q_{0}^{2}\right) k_{2}^{[\mu} k_{1}^{\nu]}-q_{0}^{2} k_{3}^{[\mu} k_{0}^{\nu]}+\right.
\end{gathered}
$$

\footnotetext{
${ }^{9}$ The symmetrization symbols imply adding the permutations required for complete symmetry. Thus for eg. $k_{1}^{\mu} k_{0}^{\nu} k_{0}^{\rho}$ requires three terms whereas $k_{2}^{\mu} k_{1}^{\nu} k_{0}^{\rho}$ requires six terms.
} 


$$
\begin{aligned}
& \left.q_{0} q_{2} k_{1}^{[\mu} k_{0}^{\nu]}-k_{0} \cdot k_{1}\left(k_{1}^{\mu} k_{1}^{\nu}+k_{2}^{\mu} k_{0}^{\nu}\right)-k_{0} \cdot k_{2} k_{0}^{\mu} k_{0}^{\nu}+k_{1} \cdot k_{1} k_{0}^{(\mu} k_{0}^{\nu)}+3 q_{3} q_{0} k_{0}^{\mu} k_{0}^{\nu}\right]+ \\
& \mathbf{Y}_{\mathbf{1}}^{\mu} \mathbf{Y}_{\mathbf{1}}^{\nu} \mathbf{Y}_{\mathbf{1}}^{\rho}\left[\left(k_{0}^{2}+q_{0}^{2}\right) \frac{k_{1}^{\mu} k_{1}^{\nu} k_{1}^{\rho}}{3 !}-k_{0} \cdot k_{1} \frac{k_{1}^{(\mu} k_{1}^{\nu} k_{0}^{\rho)}}{3 !}-q_{0}^{2} \frac{k_{2}^{(\mu} k_{1}^{\nu} k_{0}^{\rho)}}{12}+k_{1} \cdot k_{1} \frac{k_{1}^{(\mu} k_{0}^{\nu} k_{0}^{\rho)}}{3 !}+q_{0}^{2} \frac{k_{3}^{(\mu} k_{0}^{\nu} k_{0}^{\rho)}}{3 !}\right]=0
\end{aligned}
$$

The tracelessness condition becomes $\lambda_{1} k_{1} \cdot k_{1}+\lambda_{1} q_{1} q_{1}=\lambda_{1} k_{1} \cdot k_{1}+\lambda_{3} q_{0}^{2}=$ 0 and is required for gauge invariance of the above equations. The gauge transformations include the ones in (3.3.51).

\subsubsection{Quadratic Terms}

The first step is to find combinations of variables such that their gauge variations are derivatives of the lower level terms. We write the Level 3 term $k_{3}^{\mu} Y_{3}^{\mu}$ as

$$
K_{3}^{\mu} \frac{\partial Y^{\mu}}{\partial x_{3}}+K_{21}^{\mu} \frac{\partial^{2} Y^{\mu}}{\partial x_{2} \partial x_{1}}+K_{111}^{\mu} \frac{\partial^{3} Y^{\mu}}{\partial x_{1}^{3}}
$$

whose gauge variations are

$$
\delta K_{3}^{\mu}=\lambda_{3} k_{0}^{\mu} \quad, \quad \delta K_{21}^{\mu}=\lambda_{2} k_{1}^{\mu}+\lambda_{1} Q_{2} k_{0}^{\mu} \quad, \quad \delta K_{111}^{\mu}=\lambda_{1} K_{11}^{\mu}
$$

This gives us:

$$
\begin{gathered}
\delta\left(K_{3}^{\mu} \frac{\partial Y^{\mu}}{\partial x_{3}}\right)=\lambda_{3} \frac{\partial}{\partial x_{3}}\left(k_{0} \cdot Y\right), \delta\left(K_{21}^{\mu} \frac{\partial^{2} Y^{\mu}}{\partial x_{2} \partial x_{1}}\right)=\lambda_{2} \frac{\partial}{\partial x_{2}}\left(k_{1} \cdot Y_{1}\right)+\lambda_{1} \frac{\partial}{\partial x_{1}}\left(Q_{2} k_{0} \cdot Y_{2}\right) \\
\delta\left(K_{111}^{\mu} \frac{\partial^{3} Y^{\mu}}{\partial x_{1}^{3}}\right)=\lambda_{1} \frac{\partial}{\partial x_{1}}\left(K_{11} \cdot \frac{\partial^{2} Y}{\partial x_{1}^{2}}\right)
\end{gathered}
$$

We give the solution below:

$$
\begin{gathered}
q_{0} K_{3}^{\mu}=\frac{1}{2}\left[q_{0} k_{3}^{\mu}-\left(q_{1} k_{2}^{\mu}+q_{2} k_{1}^{\mu}-\frac{q_{1}^{2} k_{1}^{\mu}}{q_{0}}+\frac{q_{1}^{3} k_{0}^{\mu}}{3 q_{0}^{2}}-q_{3} k_{0}^{\mu}\right)\right] \\
q_{0} K_{21}^{\mu}=q_{2} k_{1}^{\mu}-\frac{q_{1}^{2}}{2 q_{0}} k_{1}^{\mu} \\
q_{0} K_{111}^{\mu}=\frac{1}{2}\left(q_{0} k_{3}^{\mu}+q_{1} k_{2}^{\mu}-q_{2} k_{1}^{\mu}\right)-\left(\frac{q_{3}}{2}-\frac{q_{1}^{3}}{6 q_{0}^{2}}\right) k_{0}^{\mu}
\end{gathered}
$$

Note that the sum of the three is $q_{0} k_{3}^{\mu}$ as it should be.

We use the above to write the Level 3 Lagrangian, $L$ :

$$
L=\left[i K_{3}^{\mu} Y_{3}^{\mu}+i K_{21}^{\mu} \frac{\partial^{2} Y^{\mu}}{\partial x_{2} \partial x_{1}}+i K_{111}^{\mu} \frac{\partial^{3} Y^{\mu}}{\partial x_{1}^{3}}-K_{11}^{\mu} k_{1}^{\nu} \frac{\partial^{2} Y^{\mu}}{\partial x_{1}^{2}} \frac{\partial Y^{\nu}}{\partial x_{1}}\right.
$$




$$
\left.-Q_{2} k_{0}^{\mu} k_{1}^{\nu} \frac{\partial Y^{\mu}}{\partial x_{2}} \frac{\partial Y^{\nu}}{\partial x_{1}}-\frac{i k_{1}^{\mu} k_{1}^{\nu} k_{1}^{\rho}}{3 !} Y_{1}^{\mu} Y_{1}^{\nu} Y_{1}^{\rho}\right] e^{i k_{0} Y}
$$

The quadratic term calculated in Appendix A is written in terms of $L(z)$ defined below:

$$
L_{3}^{\mu}(z) \equiv\left[V_{3}^{\mu \nu} Y_{3}^{\nu}(z)+V_{21}^{\mu \rho \sigma} Y_{2}^{\rho}(z) Y_{1}^{\sigma}(z)+V_{111}^{\mu \lambda \rho \sigma} Y_{1}^{\lambda}(z) Y_{1}^{\rho}(z) Y_{1}^{\sigma}(z)\right] e^{i k_{0} \cdot Y(z)}
$$

where

$$
\begin{aligned}
& V_{3}^{\mu \rho}=-k_{0}^{\mu}\left[K_{3}^{\rho}+K_{21}^{\rho}+K_{111}^{\rho}\right]+k_{1}^{\mu}\left[K_{11}^{\rho}+Q_{2} k_{0}^{\rho}\right]+k_{0}^{\mu} Q_{2} k_{1}^{\rho}-K_{11}^{\mu} k_{1}^{\rho}-K_{21}^{\mu} k_{0}^{\rho}+K_{111}^{\mu} k_{0}^{\rho}+K_{3}^{\mu} k_{0}^{\rho} \\
& V_{21}^{\mu \rho \sigma}=i\left[-k_{0}^{\mu} K_{11}^{\rho} k_{1}^{\rho}+k_{1}^{\mu} K_{11}^{\rho} k_{0}^{\sigma}+k_{1}^{\mu} Q_{2} k_{0}^{\rho} k_{0}^{\sigma}+k_{1}^{\mu} k_{1}^{\rho} k_{1}^{\sigma}-2 K_{11}^{\mu} k_{1}^{\rho} k_{0}^{\sigma}-K_{11}^{\mu} k_{0}^{\rho} k_{1}^{\sigma}-K_{21}^{\mu} k_{0}^{\rho} k_{0}^{\sigma}+3 K_{111}^{\mu} k_{0}^{\rho} k_{0}^{\sigma}\right] \\
& V_{111}^{\mu \lambda \rho \sigma}=\frac{1}{3 !} k_{0}^{\mu} k_{1}^{\lambda} k_{1}^{\rho} k_{1}^{\sigma}-\frac{1}{3 !} k_{1}^{\mu} k_{1}^{(\lambda} k_{1}^{\rho} k_{0}^{\sigma)}+\frac{1}{3} K_{11}^{\mu} k_{1}^{(\lambda} k_{0}^{\rho} k_{0}^{\sigma)}-K_{111}^{\mu} k_{0}^{\lambda} k_{0}^{\rho} k_{0}^{\sigma}
\end{aligned}
$$

$L_{3}^{\mu}(z)$ is a gauge invariant field strength for the massive level 3 fields. Note that the non-zero mass $\left(q_{0}\right)$ is crucial for being able to construct such an object.

We can now eliminate $q_{1}$ as before using (3.3.50). After eliminating $q_{1}$ they become:

$$
\begin{gathered}
q_{0} K_{3}^{\mu}=\frac{q_{3}}{3} k_{0}^{\mu} \\
q_{0} K_{111}^{\mu}=\frac{3}{2} k_{3}^{\mu} q_{0}-q_{2} k_{1}^{\mu}-\frac{q_{3}}{3} k_{0}^{\mu} \\
q_{0} K_{21}^{\mu}=\frac{1}{2}\left(2 q_{2} k_{1}^{\mu}-q_{0} k_{3}^{\mu}\right)
\end{gathered}
$$

The quadratic term can thus be written in a manifestly gauge invariant way as:

$$
\iint d z_{A} d z_{B} \dot{G}\left(z_{A}, z_{B}\right)\left(L_{1}^{\mu}\left(z_{A}\right)+L_{2}^{\mu}\left(z_{A}\right)+L_{3}^{\mu}\left(z_{A}\right)\right)\left(L_{1}^{\mu}\left(z_{B}\right)+L_{2}^{\mu}\left(z_{B}\right)+L_{3}^{\mu}\left(z_{B}\right)\right)
$$

where we have included all the fields from lower levels10. Once we have a gauge invariant equation we can set $x_{n}=0$ and these vertex operators reduce to standard ones. An OPE has to be then performed in the same way as was done for level 2 before we can combine this with the linear term. As mentioned earlier, it is very interesting that the gauge transformation is the same linear transformation of the free theory.

As in the case of level 2 these equations can be converted to space time form after the OPE's are performed. However since the result is not particularly illuminating we do not do it here. We hasten to add that the method while tedious is quite straightforward as we have seen in the level 2 case.

\footnotetext{
${ }^{10}$ Except for the tachyon.
} 


\subsection{Extension to Level 4}

The extension to level 4 is outlined to illustrate the general pattern. In practice the algebra may be tedious and has not been attempted. The pattern is easy to easy once we list the operators and gauge transformation at each level:

\section{Level 0}

$$
k_{0} \cdot Y
$$

Level 1

$$
k_{1}^{\mu} \frac{\partial Y}{\partial x_{1}} \quad \rightarrow \lambda_{1} \frac{\partial\left(k_{0} . Y\right)}{\partial x_{1}}
$$

Level 2

$$
\begin{aligned}
& K_{11} \frac{\partial^{2} Y}{\partial x_{1}^{2}} \rightarrow \lambda_{1} \frac{\partial}{\partial x_{1}}\left(k_{1} \frac{\partial Y}{\partial x_{1}}\right) \\
& +Q_{2} k_{0} \frac{\partial Y}{\partial x_{2}} \rightarrow \lambda_{2} \frac{\partial}{\partial x_{2}}\left(k_{0} Y\right)
\end{aligned}
$$

Level 3

$$
\begin{gathered}
K_{3} \frac{\partial Y}{\partial x_{3}} \rightarrow \lambda_{3} \frac{\partial}{\partial x_{3}}\left(k_{0} Y\right) \\
+K_{21} \frac{\partial^{2} Y}{\partial x_{1} \partial x_{2}} \quad \rightarrow \lambda_{2} \frac{\partial}{\partial x_{2}}\left(k_{1} \frac{\partial Y}{\partial x_{2}}\right)+\lambda_{1} \frac{\partial}{\partial x_{1}}\left(Q_{2} k_{0} \frac{\partial Y}{\partial x_{2}}\right) \\
+K_{111} \frac{\partial^{3} Y}{\partial x_{1}^{3}} \quad \rightarrow \lambda_{1} \frac{\partial}{\partial x_{1}}\left(K_{11} \frac{\partial^{2} Y}{\partial x_{1}^{2}}\right)
\end{gathered}
$$

From the above pattern for level 4 we need to find:

Level 4

$$
\begin{gathered}
K_{4} \frac{\partial Y}{\partial x_{4}} \rightarrow \lambda_{4} \frac{\partial}{\partial x_{4}}\left(k_{0} Y\right) \\
+K_{31} \frac{\partial^{2} Y}{\partial x_{3} \partial x_{1}} \rightarrow \lambda_{3} \frac{\partial}{\partial x_{3}}\left(k_{1} \frac{\partial Y}{\partial x_{1}}\right)+\lambda_{1} \frac{\partial}{\partial x_{1}}\left(K_{3} \frac{\partial Y}{\partial x_{3}}\right) \\
+K_{22} \frac{\partial^{2} Y}{\partial x_{2}^{2}} \rightarrow \lambda_{2} \frac{\partial}{\partial x_{2}}\left(Q_{2} k_{0} \frac{\partial Y}{\partial x_{2}}\right) \\
+K_{211} \frac{\partial^{3} Y}{\partial x_{2} \partial x_{1}^{2}} \rightarrow \lambda_{2} \frac{\partial}{\partial x_{2}} K_{11} \frac{\partial^{2} Y}{\partial x_{1}^{2}}+\lambda_{1} \frac{\partial}{\partial x_{1}}\left(K_{21} \frac{\partial^{2} Y}{\partial x_{2} \partial x_{1}}\right) \\
+K_{1111} \frac{\partial^{4} Y}{\partial x_{1}^{4}} \rightarrow \lambda_{1} \frac{\partial}{\partial x_{1}}\left(K_{111} \frac{\partial^{3} Y}{\partial x_{1}^{3}}\right)
\end{gathered}
$$


Thus we need to find combinations of the $k_{n}$ and $q_{n}$ such that

$$
\begin{gathered}
\delta K_{4}^{\mu}=\lambda_{4} k_{0}^{\mu} \quad, \quad \delta K_{31}^{\mu}=\lambda_{3} k_{1}^{\mu}+\lambda_{1} K_{3}^{\mu} \quad, \quad \delta K_{22}^{\mu}=\lambda_{2} Q_{2} k_{0}^{\mu} \\
K_{211}^{\mu}=\lambda_{2} K_{11}^{\mu}+\lambda_{1} K_{21}^{\mu} \quad, \quad K_{1111}^{\mu} \rightarrow \lambda_{1} K_{111}^{\mu}
\end{gathered}
$$

\subsection{Equivalence with String Theory}

In deriving gauge invariant equations the main ingredient was the freedom to add total derivatives in $x_{n}$. One can ask whether the theory is still equivalent to string theory. Is the S-matrix defined by this theory the same as that of string theory? It has been shown that for the free theory, one can map the fields, gauge transformations and constraints to those of the old covariant formalism - for level two and three [22]. Since the interactions are generated by calculating correlation function of vertex operators and this procedure is mathematically the same in both cases, the interacting theory should give the same physical results. A formal proof of this equivalence however has not been addressed.

\section{$5 \quad$ Summary and Conclusions}

In this paper we have written down the exact renormalization group (ERG) for the world sheet action describing an open string propagating in general backgrounds. We have shown that these equation can be made invariant under space-time gauge transformations using the loop variable technique. The equations obtained are by construction quadratic in the fields and in this sense is similar to BRST string field theory. The main difference is that the gauge transformation law is unchanged by the presence of interactions. The interaction terms can be written in terms of gauge invariant field strengths for the massive fields where the fact the mass is non zero is crucial. This is

reminiscent of the Born-Infeld action for the massless vector in open string theory in which all interactions involve only the field strength. We have demonstrated this explicitly for the massive spin 2 and spin 3 fields and outlined the pattern for the next level. It is natural to conjecture that it can be done for all levels.

Since the RG method of obtaining equations of motion works for any background (i.e one does not need to perturb around a conformal background) this method is background independent. Furthermore the gauge invariance does not depend on world sheet symmetries. This means that one can easily add a UV regulator and modify the theory at intermediate 
stages of the calculation. This we also know is necessary for going off shell. This freedom also turned out to be useful for other reasons in that we chose the extra coordinate, which played a role similar to that of a bosonized ghost of string field theory, massive. This turned out to be necessary for the correlation functions to agree with those of string theory.

One should add that quite independent of string theory, (at least at the tree level) this technique gives a gauge invariant massive interacting higher spin theory in any dimension. The tachyon can also be made massive if necessary. The constraint about dimension and mass spectrum comes form requiring agreement with string theory. This presumably also ensures consistency at the loop level. If this is found to be not necessary then one can generalize to other theories.

There are many questions that need to be answered. Probably the most pressing is whether this technique can be generalized to closed strings. That would give a quadratic equation of motion, unlike string field theory, which for closed strings is non polynomial. The other pressing question is to construct an action. Another issue is to give a rigorous proof of the equivalence of the S-matrix of this theory with that of string theory.

\section{A Appendix: Level 3 Quadratic Terms}

We give the calculation of the level 3 quadratic pieces:

$$
\begin{gathered}
\frac{\partial L}{\partial Y^{\mu}}=i k_{0}^{\mu}\left[i K_{3}^{\rho} Y_{3}^{\rho}+i K_{21}^{\rho} \frac{\partial^{2} Y^{\rho}}{\partial x_{2} \partial x_{1}}+i K_{111}^{\rho} \frac{\partial^{3} Y^{\rho}}{\partial x_{1}^{3}}-K_{11}^{\rho} k_{1}^{\sigma} \frac{\partial^{2} Y^{\rho}}{\partial x_{1}^{2}} \frac{\partial Y^{\sigma}}{\partial x_{1}}\right. \\
\left.-Q_{2} k_{0}^{\rho} k_{1}^{\sigma} \frac{\partial Y^{\rho}}{\partial x_{2}} \frac{\partial Y^{\sigma}}{\partial x_{1}}-\frac{\left(i k_{1} \cdot Y_{1}\right)^{3}}{3 !}\right] e^{i k_{0} Y} \\
\partial_{x_{1}} \frac{\partial L}{\partial Y_{1}^{\mu}}=\left[-K_{11}^{\rho} \frac{\partial^{3} Y^{\rho}}{\partial x_{1}^{3}} k_{1}^{\mu}-K_{11}^{\rho} \frac{\partial^{2} Y^{\rho}}{\partial x_{1}^{2}} k_{1}^{\mu}\left(i k_{0} \cdot Y\right)-\right. \\
\left.Q_{2} k_{0}^{\rho} \frac{\partial^{2} Y^{\rho}}{\partial x_{2} \partial x_{1}} k_{1}^{\mu}-Q_{2} k_{0} \cdot Y_{2} k_{1}^{\mu}\left(i k_{0} \cdot Y_{1}\right)-i k_{1}^{\mu} k_{1} \cdot Y_{2} k_{1} \cdot Y_{1}-\frac{i k_{1}^{\mu}}{2}\left(k_{1} \cdot Y_{1}\right)^{2}\left(i k_{0} \cdot Y_{1}\right)\right] e^{i k_{0} Y} \\
\partial_{x_{2}} \frac{\partial L}{\partial Y_{2}^{\mu}}=-\left[Q_{2} k_{0}^{\mu} k_{1} \cdot Y_{3}+Q_{2} k_{0}^{\mu}\left(k_{1} \cdot Y_{1}\right)\left(i k_{0} \cdot Y_{2}\right)\right] e^{i k_{0} Y} \\
\partial_{x_{1}}^{2} \frac{\partial L}{\partial\left(\frac{\partial^{2} Y^{\mu}}{\partial x_{1}^{2}}\right)}=-\left[K_{11}^{\mu} k_{1} \cdot Y_{3}+2 K_{11}^{\mu} k_{1} \cdot Y_{2} i k_{0} \cdot Y_{1}+K_{11}^{\mu} k_{1} \cdot Y_{1}\left[i k_{0} \cdot Y_{2}+\left(i k_{0} \cdot Y_{1}\right)^{2}\right]\right] e^{i k_{0} Y} \\
\partial_{x_{1}} \partial_{x_{2}} \frac{\partial L}{\partial\left(\frac{\partial^{2} Y^{\mu}}{\partial x_{1} \partial x_{2}}\right)}=\left[i K_{21}^{\mu} i k_{0} \cdot Y_{3}+i K_{111}^{\mu} i k_{0} \cdot Y_{2} i k_{0} \cdot Y_{1}\right] e^{i k_{0} Y}
\end{gathered}
$$




$$
\begin{gathered}
\partial_{x_{1}}^{3} \frac{\partial L}{\partial\left(\frac{\partial^{3} Y^{\mu}}{\partial x_{1}^{3}}\right)}=i K_{111}^{\mu}\left[i k_{0} \cdot Y_{3}+3 i k_{0} \cdot Y_{2} i k_{0} \cdot Y_{1}+\left(i k_{0} \cdot Y_{1}\right)^{3}\right] e^{i k_{0} Y} \\
\partial_{x_{3}} \frac{\partial L}{\partial Y_{3}^{\mu}}=i K_{3}^{\mu} i k_{0} \cdot Y_{3} e^{i k_{0} Y}
\end{gathered}
$$

Adding these terms (with appropriate signs 11) we get

$$
L_{3}^{\mu}(z) \equiv\left[V_{3}^{\mu \nu} Y_{3}^{\nu}(z)+V_{21}^{\mu \rho \sigma} Y_{2}^{\rho}(z) Y_{1}^{\sigma}(z)+V_{111}^{\mu \lambda \rho \sigma} Y_{1}^{\lambda}(z) Y_{1}^{\rho}(z) Y_{1}^{\sigma}(z)\right] e^{i k_{0} . Y(z)}
$$

where

$$
\begin{aligned}
& V_{3}^{\mu \rho}=-k_{0}^{\mu}\left[K_{3}^{\rho}+K_{21}^{\rho}+K_{111}^{\rho}\right]+k_{1}^{\mu}\left[K_{11}^{\rho}+Q_{2} k_{0}^{\rho}\right]+k_{0}^{\mu} Q_{2} k_{1}^{\rho}-K_{11}^{\mu} k_{1}^{\rho}-K_{21}^{\mu} k_{0}^{\rho}+K_{111}^{\mu} k_{0}^{\rho}+K_{3}^{\mu} k_{0}^{\rho} \\
& V_{21}^{\mu \rho \sigma}=i\left[-k_{0}^{\mu} K_{11}^{\rho} k_{1}^{\rho}+k_{1}^{\mu} K_{11}^{\rho} k_{0}^{\sigma}+k_{1}^{\mu} Q_{2} k_{0}^{\rho} k_{0}^{\sigma}+k_{1}^{\mu} k_{1}^{\rho} k_{1}^{\sigma}-2 K_{11}^{\mu} k_{1}^{\rho} k_{0}^{\sigma}-K_{11}^{\mu} k_{0}^{\rho} k_{1}^{\sigma}-K_{21}^{\mu} k_{0}^{\rho} k_{0}^{\sigma}+3 K_{111}^{\mu} k_{0}^{\rho} k_{0}^{\sigma}\right] \\
& V_{111}^{\mu \lambda \rho \sigma}=\frac{1}{3 !} k_{0}^{\mu} k_{1}^{\lambda} k_{1}^{\rho} k_{1}^{\sigma}-\frac{1}{3 !} k_{1}^{\mu} k_{1}^{(\lambda} k_{1}^{\rho} k_{0}^{\sigma)}+\frac{1}{3} K_{11}^{\mu} k_{1}^{(\lambda} k_{0}^{\rho} k_{0}^{\sigma)}-K_{111}^{\mu} k_{0}^{\lambda} k_{0}^{\rho} k_{0}^{\sigma}
\end{aligned}
$$

\section{B Appendix: Operator Product Expansion}

We work out some of the operator product expansions that are needed. The general master formula can be easily written in terms of loop variables:

$$
\begin{aligned}
& : e^{\left(i k_{0} . Y+i k_{1} \cdot Y_{1}+\ldots+i k_{n} . Y_{n}\right)\left(z_{1}\right)}:: e^{\left(i p_{0} \cdot Y+i p_{1} . Y_{1}+\ldots+i p_{m} . Y_{m}\right)\left(z_{2}\right)}:= \\
& \quad e^{-\sum_{n, m} k_{n} \cdot p_{m} G_{n, m}\left(z_{1}-z_{2}\right)} \\
& : e^{\left(i k_{0} . Y+i k_{1} . Y_{1}+\ldots+i k_{n} . Y_{n}\right)\left(z_{1}\right)+\left(i p_{0} . Y+i p_{1} \cdot Y_{1}+\ldots+i p_{m} . Y_{m}\right)\left(z_{2}\right)}:
\end{aligned}
$$

where $G_{n, m}\left(z_{1}-z_{2}\right)=\left\langle Y_{n}\left(z_{1}\right) Y_{m}\left(z_{2}\right)\right\rangle$.

We can extract from (B.1) terms multilinear in $k_{i}$ and $p_{j}$ to extract OPE's of the usual vertex operators.

The following gives the general normal ordering of vertex operators at one point:

$e^{\left(i k_{0} . Y+i k_{1} \cdot Y_{1}+\ldots+i k_{n} . Y_{n}\right)\left(z_{1}\right)}=e^{-\frac{1}{2} \sum_{n, m} k_{n} \cdot k_{m} G_{n, m}\left(z_{1}, z_{1}\right)}: e^{\left(i k_{0} \cdot Y+i k_{1} \cdot Y_{1}+\ldots+i k_{n} . Y_{n}\right)\left(z_{1}\right)}:$

Thus typically one uses (B.2) followed by (B.1) if the vertex operators are not normal ordered to begin with.

\footnotetext{
${ }^{11}$ Terms generated by $\partial_{x_{n}}$ come with minus signs (as in the usual Lagrange's equations), Terms with $\partial_{x_{n}} \partial_{x_{m}}$ come with a plus sign, and $\partial_{x_{1}}^{3}$ comes with a minus sign
} 


\section{B.1 OPE of level 1 vector vertex operators:}

The bilinear in $k_{1} p_{1}$ gives us the OPE between $i: k_{1} \cdot Y_{1} e^{i k_{0} \cdot Y\left(z_{1}\right)}:$ and $i: p_{1} . Y_{1} e^{i p_{0} . Y\left(z_{2}\right)}:$. (Thus in the situation of interest to us $k_{1}^{\mu} Y_{1}^{\mu}$ would be replaced by $\left.F^{\nu \mu}\left(k_{0}\right) Y_{1}^{\mu}\right)$.

We get the following four terms: (We have suppressed the argument $\left(z_{1}-z_{2}\right)$ of the Greens function in the equations below)

$$
\begin{gathered}
\text { i) } \quad: i k_{1} \cdot Y_{1}\left(z_{1}\right) i p_{1} \cdot Y_{1}\left(z_{2}\right) e^{i k_{0} \cdot Y\left(z_{1}\right)+i p_{0} \cdot Y\left(z_{2}\right)}: \\
\text { ii) }-k_{0} \cdot p_{1} G_{0,1}: i k_{1} \cdot Y_{1}\left(z_{1}\right) e^{i k_{0} \cdot Y\left(z_{1}\right)+i p_{0} \cdot Y\left(z_{2}\right)}: \\
\text { iii) } \quad-k_{1} \cdot p_{0} G_{1,0}: i p_{1} \cdot Y_{1}\left(z_{2}\right) e^{i k_{0} \cdot Y\left(z_{1}\right)+i p_{0} \cdot Y\left(z_{2}\right)}: \\
\text { iv) } \quad k_{0} \cdot p_{1} p_{0} \cdot k_{1}\left[G_{0,1} G_{1,0}\right]: e^{i k_{0} \cdot Y\left(z_{1}\right)+i p_{0} \cdot Y\left(z_{2}\right)}: \\
\text { v) } \quad-k_{1} \cdot p_{1} G_{1,1}: e^{i k_{0} \cdot Y\left(z_{1}\right)+i p_{0} \cdot Y\left(z_{2}\right)}:
\end{gathered}
$$

Now a Taylor expansion about $z_{1}$ can be performed to extract various contributions. The contribution to the level 1 vertex operator $Y_{1}^{\mu}\left(z_{1}\right) e^{i k_{0} . Y\left(z_{1}\right)}$ is:

$$
\begin{gathered}
{\left[-i k_{0} \cdot p_{1} k_{1}^{\mu} G_{0,1}-i p_{0} \cdot k_{1} p_{1}^{\mu} G_{1,0}+i\left(z_{2}-z_{1}\right) G_{0,1} G_{1,0} k_{0} \cdot p_{1} k_{1} \cdot p_{0} p_{0}^{\mu}\right.} \\
\left.-\left(z_{2}-z_{1}\right) k_{1} \cdot p_{1} G_{1,1} i p_{0}^{\mu}\right]: Y_{1}^{\mu} e^{i\left(k_{0}+p_{0}\right) \cdot Y\left(z_{1}\right)}:
\end{gathered}
$$

Similarly one can extract the contribution to the level 2 vertex operator $Y_{1}^{\mu} Y_{1}^{\nu} e^{i k_{0} . Y\left(z_{1}\right)}$. The result is:

$$
\begin{gathered}
{\left[k_{1}^{\mu} p_{1}^{\nu}+\left(z_{2}-z_{1}\right) k_{0} \cdot p_{1} G_{0,1} k_{1}^{\mu} p_{0}^{\nu}+\left(z_{2}-z_{1}\right) p_{0} \cdot k_{1} p_{1}^{\mu} k_{0}^{\nu} G_{1,0}+\right.} \\
\left.-\frac{\left(z_{2}-z_{1}\right)^{2}}{2} G_{0,1} G_{1,0} k_{1} \cdot p_{0} p_{1} \cdot k_{0} p_{0}^{\mu} p_{0}^{\nu}+\frac{\left(z_{2}-z_{1}\right)^{2}}{2} G_{1,1} k_{1} \cdot p_{1} p_{0}^{\mu} p_{0}^{\nu}\right]: Y_{1}^{\mu} Y_{1}^{\nu} e^{i\left(k_{0}+p_{0}\right) \cdot Y\left(z_{1}\right)}:
\end{gathered}
$$

\section{B.2 OPE of level 1 and level 2}

We have to pick terms proportional to $k_{1}^{\mu} k_{1}^{\nu} p_{1}^{\rho}$ to get the OPE of $-\frac{1}{2} k_{1} \cdot Y_{1} k_{1} \cdot Y_{1} e^{i k_{0} . Y}$ and $i p_{1} . Y_{1} e^{i p_{0} . Y}$ :

The contribution to level 1 is

$$
\begin{gathered}
{\left[\frac{\left(i k_{1} \cdot p_{0} G_{1,0}\right)^{2}}{2} p_{1}^{\mu}+i k_{1} \cdot p_{0} G_{1,0} p_{1} \cdot k_{1} G_{0,1} k_{1}^{\mu}+i\left(z_{2}-z_{1}\right) \frac{\left(k_{1} \cdot p_{0} G_{1,0}\right)^{2}}{2} p_{1} \cdot k_{0} G_{0,1} p_{0}^{\mu}\right.} \\
\left.\quad-i k_{1} \cdot p_{1} G_{1,1} k_{1}^{\mu}+i\left(z_{2}-z_{1}\right) k_{1} \cdot p_{1} G_{1,1} k_{1} \cdot p_{0} G_{1,0} p_{0}^{\mu}\right]: Y_{1}^{\mu} e^{i\left(k_{0}+p_{0}\right) \cdot Y\left(z_{1}\right)}:
\end{gathered}
$$


The contribution to level $2 Y_{1}^{\mu} Y_{1}^{\nu}$ is

$$
\begin{gathered}
{\left[k_{1} \cdot p_{0} G_{1,0} k_{1}^{\mu} p_{1}^{\nu}-\left(z_{2}-z_{1}\right) \frac{\left(k_{1} \cdot p_{0} G_{1,0}\right)^{2}}{2} p_{1}^{m} u p_{0}^{n} u+\frac{1}{2} p_{1} \cdot k_{0} G_{0,1} k_{1}^{\mu} k_{1}^{\nu}-\right.} \\
\left(z_{2}-z_{1}\right) k_{1} \cdot p_{0} p_{1} \cdot k_{0} G_{0,1} G_{1,0} k_{1}^{\mu} p_{0}^{\nu}-\frac{\left(z_{2}-z_{1}\right)^{2}}{2} k_{1} \cdot p_{1} G_{1,1} k_{1} \cdot p_{0} G_{1,0} p_{0}^{\mu} p_{0}^{\nu} \\
\left.\left(z_{2}-z_{1}\right)^{2} \frac{\left(k_{1} \cdot p_{0} G_{1,0}\right)^{2}}{4} p_{1} \cdot k_{0} G_{0,1} p_{0}^{\mu} p_{0}^{\nu}+\left(z_{2}-z_{1}\right) k_{1} \cdot p_{1} G_{1,1} k_{1}^{\mu} p_{0}^{\nu}\right]: Y_{1}^{\mu} Y_{1}^{\nu} e^{i\left(p_{0}+k_{0}\right) \cdot Y\left(z_{1}\right)}:
\end{gathered}
$$

\section{B.3 OPE of level 2 and level 2}

We obtain the OPE of $: \frac{1}{2}\left(k_{1} \cdot Y_{1}\right)^{2} e^{i k_{0} . Y\left(z_{1}\right)}:$ and $: \frac{1}{2}\left(p_{1} \cdot Y_{1}\right)^{2} e^{i p_{0} . Y\left(z_{2}\right)}:$.

We have to pick terms proportional to $k_{1}^{\mu} k_{1}^{\nu} p_{1}^{\rho} p_{1}^{\sigma}$.

We give the contribution to level 1 :

$$
\begin{gathered}
{\left[-i \frac{\left(k_{1} \cdot p_{0} G_{1,0}\right)^{2}}{2} p_{1} \cdot k_{0} G_{0,1} p_{1}^{\mu}-i \frac{\left(p_{1} \cdot k_{0} G_{0,1}\right)^{2}}{2} p_{0} \cdot k_{1} G_{0,1} k_{1}^{\mu}+\right.} \\
\frac{\left(k_{1} \cdot p_{0} G_{1,0}\right)^{2}}{2} \frac{\left(p_{1} \cdot k_{0} G_{0,1}\right)^{2}}{2} p_{0}^{\mu}\left(z_{2}-z_{1}\right)+\left(z_{2}-z_{1}\right) \frac{\left(k_{1} \cdot p_{1} G_{1,1}\right)^{2}}{2} i p_{0}^{\mu}+i k_{1} \cdot p_{1} G_{1,1} k_{1} \cdot p_{0} G_{1,0} i p_{1}^{\mu} \\
\left.+i k_{1} \cdot p_{1} G_{1,1} k_{0} \cdot p_{1} G_{0,1} i k_{1}^{\mu}-k_{1} \cdot p_{1} G_{1,1} p_{1} \cdot k_{0} G_{0,1} k_{1} \cdot p_{0} G_{1,0}\left(z_{2}-z_{1}\right) p_{0}^{\mu}\right]: Y_{1}^{\mu} e^{i\left(k_{0}+p_{0}\right) \cdot Y\left(z_{1}\right)}:
\end{gathered}
$$

Similarly the contribution to $Y_{1}^{\mu} Y_{1}^{\nu}$ consists of nine terms. We do not list them here.

\section{References}

[1] C. Lovelace, Phys. Lett. B135,75 (1984).

[2] C. Callan, D. Friedan, E. Martinec and M. Perry, Nucl. Phys. B262,593 (1985).

[3] A. Sen, Phys. Rev. D32,2102 (1985).

[4] E. Fradkin and A.A. Tseytlin, Phys. Lett. B151,316 (1985).

[5] C. Callan and Z. Gan, Nucl. Phys. B272, 647 (1987)

[6] S. Das and B. Sathiapalan, Phys. Rev. Lett. B183,65 (1985).

[7] B. Sathiapalan, Nucl. Phys. B294, (1987) 747.

[8] J. Hughes,J. Liu and J. Polchinski, Nucl. Phys. B316 (1989). 
[9] T. Banks and E. Martinec, Nucl. Phys. B294, 733 (1987).

[10] V.A. Kostelecky, M.J. Perry, and R. Potting, Phys. Rev. Lett. 84 (2000) 4541, hepth/9912243.

[11] I.L. Buchbinder, O.M.Gitman,V.A. Krykhtin and Y.D. Pershin, hepth/9910188, Nucl. Phys. B584 (2000) 615.

[12] A. Abouelsaood, C. G. Callan, C. R. Nappi and S. A. Yost, Nucl. Phys. B280,(1989) 599.

[13] A. Polyakov, "Gauge Fields and Strings", Harwood Academic Publishers, New York, 1987.

[14] A.A. Tseytlin, Int. J. Mod. Phys. A4:4249 (1989) and references therein.

[15] B. Sathiapalan, Nucl. Phys. B326 (1989)376.

[16] C. Fronsdal, Phys. Rev D18 (1978) 3624.

[17] L. P. S. Singh and C. R. Hagen, Phys. Rev D9 (1974) 898.

[18] B. Sathiapalan, hepth/0207098, Int.J.Mod. Phys. A18 (2003) 767-810.

[19] B. Sathiapalan, hep-th/0405119, Mod.Phys.Lett. A19 (2004) 2857.

[20] B. Sathiapalan, hepth/9409023, Int. J. Mod. Phys. A10 (1995) 4501.

[21] B. Sathiapalan, Phys. Lett. B201 (1988) 454.

[22] B. Sathiapalan, hep-th/0602090, Mod.Phys.Lett. A21 (2006) 863-882 .

[23] B. Sathiapalan, Mod. Phys. Lett A 19 (2007) 1701.

[24] B. Sathiapalan, JHEP 0907 (2009) 045 ; arXiv:0902.1132 [hep-th].

[25] K.G. Wilson and J. Kogut, Phys. Reports 12 , (1974)75.

[26] K.G.Wilson, Rev. Mod. Phys. 47, (1975)773.

[27] K.G. Wilson, Phs. Rev. D7 (1973)2911.

[28] J. Polchinski, Nucl. Phys. B231 (1984) 269.

[29] J. Hughes, J. Liu and J. Polchinski, Nucl.Phys.B316:15,1989.

[30] W. Siegel and B. Zwiebach, Nucl. Phys. B263 (1986) 105. 
[31] W. Siegel, Phys. Lett B149, 157; 162 (1984);B151 391;396 (1985).

[32] E. Witten, Nucl. Phys B268, (1986) 513.

[33] B. Zwiebach, Nucl.Phys.B390:33,1993, hep-th/9206084.

[34] E. Witten, hepth/9208027, Phys. Rev D 46 (1992) 5467; hepth/9210065, Phys. Rev. D 47 (9193) 3405.

[35] K. Li and E. Witten, hepth/9303067, Phys. Rev. D 48 (1993) 7297.

[36] S. Shatashvili, hepth/9303143, Phys. Lett B311 (1993)83; hepth/9311177.

[37] D. Kutasov, M. Marino, and G. Moore, hepth/0009148.

[38] C. Bagnuls and C. Bervillier, hep-th/0101110, Int.J.Mod.Phys.A16:1825,2001.

[39] C. Bagnuls and C. Bervillier, hep-th/0002034, Phys.Rept.348:91,2001.

[40] H. Sonoda, hep-th/0612294 Phys.Rev.D67:065011,2003, hep-th/0212302.

[41] Y. Igarashi, K. Itoh and H. Sonoda, arXiv:0704.2349 [hep-th].

[42] B. Sathiapalan, Mod.Phys.Lett.A22:107-118,2007, hep-th/0612069

[43] K. Symanzik, Nucl.Phys.B226:187,1983.

[44] P. Hasenfratz and F. Niedermayer , Nucl.Phys.B414:785-814,1994; hep-lat/9308004.

[45] B. Sathiapalan, hepth/9509097; Intl. J. Mod. Phys. A 11 (1996) 2887.

[46] "Current Physics Sources and Comments" - Vol 2 - "Finite Size Scaling" edited by J. Cardy, North-Holland, 1988. 